Check for updates

Cite this: Nanoscale Adv., 2019, 1, 4025

\section{Large-scale efficient water harvesting using bioinspired micro-patterned copper oxide nanoneedle surfaces and guided droplet transport $\uparrow$}

\author{
Vipul Sharma, (D) ${ }^{a}$ Kyriacos Yiannacou, ${ }^{a}$ Markus Karjalainen, ${ }^{a}$ Kimmo Lahtonen, (D) ${ }^{b}$ \\ Mika Valden ${ }^{\text {b }}$ and Veikko Sariola*a
}

\begin{abstract}
As the Earth's atmosphere contains an abundant amount of water as vapors, a device which can capture a fraction of this water could be a cost-effective and practical way of solving the water crisis. There are many biological surfaces found in nature which display unique wettability due to the presence of hierarchical micro-nanostructures and play a major role in water deposition. Inspired by these biological microstructures, we present a large scale, facile and cost-effective method to fabricate water-harvesting functional surfaces consisting of high-density copper oxide nanoneedles. A controlled chemical oxidation approach on copper surfaces was employed to fabricate nanoneedles with controlled morphology, assisted by bisulfate ion adsorption on the surface. The fabricated surfaces with nanoneedles displayed high wettability and excellent fog harvesting capability. Furthermore, when the fabricated nanoneedles were subjected to hydrophobic coating, these were able to rapidly generate and shed coalesced droplets leading to further increase in fog harvesting efficiency. Overall, $99 \%$ and $\sim 150 \%$ increase in fog harvesting efficiency was achieved with non-coated and hydrophobic layer coated copper oxide nanoneedle surfaces respectively when compared to the control surfaces. As the transport of the harvested water is very important in any fog collection system, hydrophilic channels inspired by leaf veins were made on the surfaces via a milling technique which allowed an effective and sustainable way to transport the captured water and further enhanced the water collection efficiency by $\sim 9 \%$. The system presented in this study can provide valuable insights towards the design and fabrication of fog harvesting systems, adaptable to arid or semi-arid environmental conditions.
\end{abstract}

Received 27th June 2019

Accepted 3rd September 2019

DOI: $10.1039 /$ c9na00405j

rsc.li/nanoscale-advances will have a scarcity of water in the future. ${ }^{1-3}$ This will also cause other problems related to proper sanitation which will directly have an impact on the public health concerns around the world. ${ }^{4}$ Apart from the desalination of seawater as a solution to this problem, atmospheric water capture has been intensively researched as a potential solution for harvesting clean water in the arid and semi-arid areas..$^{5,6}$ One potential source of clean water from the atmosphere is fog, which is suspended in the air as a visible cloud of water droplets. In the recent decade, efficient fog collection capability of multiscale hierarchical surfaces in various flora and fauna has inspired researchers to develop synthetic bioinspired fog collection surfaces. ${ }^{7-15}$ Specifically, these surfaces take advantage of the combination of the unique micro and nanostructures which are bioinspired from the biological structures such as multilevel combination of micro and nanostructures of Gladiolus dalenii and microstructured slippery surfaces of the pitcher plant. ${ }^{16}$ Among the specific structures, conical spine-like structures, ${ }^{17-19}$ bumps of beetles, ${ }^{11,20}$ micro-structured spindle knot surfaces of spider silk, ${ }^{21,22}$ along with others endow the structures with water collection ability.
${ }^{a}$ Faculty of Medicine and Health Technology, Tampere University, Korkeakoulunkatu 3, 33720 Tampere, Finland. E-mail: veikko.sariola@tuni.fi

${ }^{b}$ Faculty of Engineering and Natural Sciences, Tampere University, P.O. Box 692, FI33014, Finland

$\dagger$ Electronic supplementary information (ESI) available: FESEM of the surfaces in the absence of scoring and $\mathrm{H}_{2} \mathrm{SO}_{4}$; XPS data of the bisulfate adsorption to the $\mathrm{Cu}$ surface; time lapse wettability studies on $\mathrm{CuO}$; additional contact angle data and repeatability studies. See DOI: 10.1039/c9na00405j 
The adapted ability of biological surfaces to harvest atmospheric water is attributed to patterns of hydrophilic and hydrophobic areas. The water droplets in the fog are captured by the hydrophilic areas and get transported with the help of hydrophobic areas. ${ }^{23,24}$ Many bioinspired and biomimetic surfaces have been developed and demonstrated to have exceptional fog harvesting capability based on the optimized combinations of super-hydrophobic and super-hydrophilic patterns having micro- and nanoscale structures. ${ }^{7,25,26}$ The most important factor here that plays a major role in efficient water collection is the type of multiscale surface morphology. For example, Cotula fallax and Cynodon dactylon are able to collect fog by their hierarchical 3D structures which provide them with a large surface area to volume ratio. ${ }^{17,27}$ In a recent report by Lu et al. ${ }^{28}$ it has been demonstrated that biomimetic PDMS film inspired by the rice leaf can facilitate the fog harvesting process by virtue of the multi-level hierarchical periodic macrogrooves covered with micro/nanostructures which aid in the anisotropic sliding wetting. Different types of fibers have also been fabricated such as polyethylene terephthalate (PET) fibers which display very good fog harvesting capability due to orientation and surface morphology. ${ }^{29}$ In addition, superhydrophilic wire-like structures and arrays composed of different materials such as zinc oxide, aluminum and copper were also reported which were efficient in droplet spreading and transport of the harvested fog. ${ }^{30-32} 3 \mathrm{D}$ nanowire-like structures based on copper oxide have also been reported where the surfaces displayed a remarkable transition in wettability upon adsorption of carbon species upon ambient exposure. ${ }^{33}$ Using different micropatterning techniques employing different metals and polymers, super-hydrophilic microstructures surrounded by superhydrophobic areas have been developed which display a range of morphologies, such as microcones, bulges, and nanowhiskers, among others. ${ }^{\mathbf{1 3 2 4 , 3 0 - 3 2 , 3 4 - 4 0}}$ These microstructured surfaces had areas with high wettability and low wettability and displayed enhanced fog harvesting efficiency ranging from $0.006 \mathrm{~g} \mathrm{~cm}^{-2} \mathrm{~h}^{-1}$ to $2.78 \mathrm{~g} \mathrm{~cm}^{-2} \mathrm{~h}^{-1}$. Therefore, the effect of hierarchical surface features and wettability on the fog harvesting capability is worth studying. We note here that the range of values reported of up to 4 orders of magnitude difference on the fog collection efficiency are strongly influenced by the fog harvesting technique and experimental conditions utilized. ${ }^{13}$

Previous studies have suggested that the variations in the Laplace pressures, which arise from the energy gradients at the surfaces because of their conical or pointed morphologies and chemical composition of materials, stimulate coalescence of small droplets condensed on the surfaces. ${ }^{\mathbf{1 8 , 1 9 , 4 1 , 4 2}}$ In most of the reported cases, deposited droplets can be spontaneously and continuously captured and transported from the tip to the base of the conical structures due to difference in the Laplace pressure. However, the difference in Laplace pressure by constraining the droplet geometry between the microstructures such as micro-pillars and micro-patterned nanowire arrays is also known to induce droplet detachment from the surface which may lead to better water collection. ${ }^{\mathbf{4 3 4 4}}$ In these cases, dewetting transition of deposited droplets occurred on microstructures with optimized spacing. The droplets migrate from the base to the top of the microstructures and it is related to the topography of the fabricated microstructures. In addition, Shanahan has also reported that in order to attain minimum surface energy, dew droplets are able to climb a conical structure which is aided by the capillary effect. ${ }^{45}$ Currently, there are not many studies which have attempted to realize fog harvesting devices with high output and the existing ones are facing challenges like high production cost, low efficiencies and most importantly scaling up.

The directional water transportability of most specific biological structures is strictly dependent on many factors such as air flow, gravitational forces, adhesion forces, etc. ${ }^{\mathbf{1 5 , 2 1 , 4 6 - 4 8}}$ These forces can hinder the fog collection cycle from restarting and raise the risk of droplet re-evaporation, either by halting the timely removal of the nucleated and grown droplet or by prematurely removing the nucleated droplets. ${ }^{49-51}$ Although the current bioinspired fog collectors are quite successful in producing a reasonable amount of water, the transport of water is still of vital importance to the removal of captured droplets. With respect to the disadvantages of gravity-driven bioinspired fog collectors at a large scale, droplet retention on the functional surfaces may suffer from severe re-evaporation under fog flow, which may have a major effect on the efficiency and the consistency of the fog collectors. ${ }^{52}$ Also, to efficiently collect the nucleated and coalesced droplets, which may travel across the surface and foul the nucleation sites by terminating the nucleation and growth process prematurely, it is important to design a surface which facilitates the directional transport.

Inspired by nature, we propose the fabrication of $\mathrm{CuO}$ nanoneedles on a large scale for potential fog harvesting application. High-density $\mathrm{CuO}$ nanoneedles were fabricated using a low cost and facile wet chemical oxidation process which can be scaled up very easily. The morphological and compositional characteristics of $\mathrm{CuO}$ nanoneedles are discussed and confirmed using field emission scanning electron microscopy (FESEM) and X-ray photoelectron spectroscopy (XPS). The shape and orientation of the nanoneedles on the surface provide unique wettability to the surfaces and enable them to capture tiny water droplets from the fog flow. As directional transportation is the key for efficient collection of water, hydrophilic channels were made on the surface via an economically viable milling technique which draws the nucleated and coalesced water from across the surface and aids in proficient water collection. The surface wetting properties of the nanoneedles were studied and their correlation with the fog collection ability was established. The underlying mechanisms governing the droplet nucleation and the dynamic transport on the surface have also been highlighted.

\section{Materials and methods}

\section{Fabrication procedures}

$\mathrm{Cu}$ plates having dimensions $150 \mathrm{~mm} \times 150 \mathrm{~mm} \times 1.5 \mathrm{~mm}$ were used in this study. These dimensions were chosen as it is easy to monitor these in fog harvesting experiments. However, 
with the current methodology, the fabrication of the bioinspired surfaces can be achieved on a much larger scale.

Millichannel fabrication. Open channels were milled using a Makrum SMX3500 mill (Tampere, Finland) which can fabricate to a maximum area of $700 \mathrm{~mm} \times 450 \mathrm{~mm}$. Channels had an approximate depth of $0.4 \mathrm{~mm}$ and a width of $1 \mathrm{~mm}$, milled in the pattern shown in Fig. 1a and b. A leaf vein-like design was adopted for the channel milling on to the surface as the design is reported to be efficient in water harvesting applications. ${ }^{53}$ The channel width and depth are adopted from the study by Mertaniemi et al. wherein the milling technique was used to fabricate channels on to hydrophobic copper surfaces for swift droplet movement. ${ }^{47}$

Nanostructure fabrication. The $\mathrm{Cu}$ plates with channels were first cleaned with acetone, ethanol and deionized water (ELGA PURELAB Option-R7) via ultra-sonication for $15 \mathrm{~min}$ in each. After being dried with high purity $\mathrm{N}_{2}$, the surface of the plates was scored gently with sandpaper ( $3 \mathrm{M}, 600$ grits) towards the direction of the channels. In the first step of fabrication of $\mathrm{CuO}$ surfaces, the scored $\mathrm{Cu}$ plates were cleaned with high purity $\mathrm{N}_{2}$ and immersed in a $0.02 \mathrm{M} \mathrm{H}_{2} \mathrm{SO}_{4}$ solution (Sigma Aldrich) for $5 \mathrm{~min}$. After this step, the $\mathrm{Cu}$ plates were transferred to a container with an aqueous solution of $1 \mathrm{wt} \% \mathrm{NaOH}$ (Sigma Aldrich) and $\left(\mathrm{NH}_{4}\right)_{2} \mathrm{~S}_{2} \mathrm{O}_{8}$ (Sigma Aldrich) in a $1: 1$ ratio at room temperature for $15 \mathrm{~min}$ with slight stirring. At the end of the reaction, the substrate was taken out of the solution, rinsed several times with ethanol first, followed by DI water, and dried with compressed $\mathrm{N}_{2}$. The plates were then dried at $150{ }^{\circ} \mathrm{C}$ for $2 \mathrm{~h}$ to complete the phase transition from a light bluish brown colored hydroxide to a dark brown colored oxide (Fig. 1) which is the surface bearing high-density $\mathrm{CuO}$ nanoneedles. To make the prepared oxide surfaces superhydrophobic, fluorosilane treatment was used to decrease the surface energy.

Hydrophobic coating procedure. The as-fabricated surfaces were placed in a sealed desiccator with few drops of $1 \mathrm{H}, 1 \mathrm{H}, 2 \mathrm{H}, 2 \mathrm{H}$-perfluorodecyltriethoxysilane (Sigma Aldrich) in it. The desiccator with the samples was then kept overnight at room temperature before the samples were taken out. The silane treated plates were then placed in the oven for 1 hour to get rid of any unattached silane on to the surface. To avoid any silane attachment into the channels, $\mathrm{Cu}$ wires soaked in $1 \%$ PVA (Sigma Aldrich) solution were carefully placed inside the channels before placing the plates in the desiccator with the silane. After the silane treatment over the plate, the $\mathrm{Cu}$ wires soaked in the PVA were carefully removed from the channels which remained hydrophilic. The samples: bare $\mathrm{Cu}$, scored $\mathrm{Cu}$ and silanized bare $\mathrm{Cu}$ and scored $\mathrm{Cu}$ were used as the control samples.

\section{Structural characterization}

X-ray photoelectron spectroscopy (XPS) and X-ray Auger electron spectroscopy (XAES) were performed utilizing non-

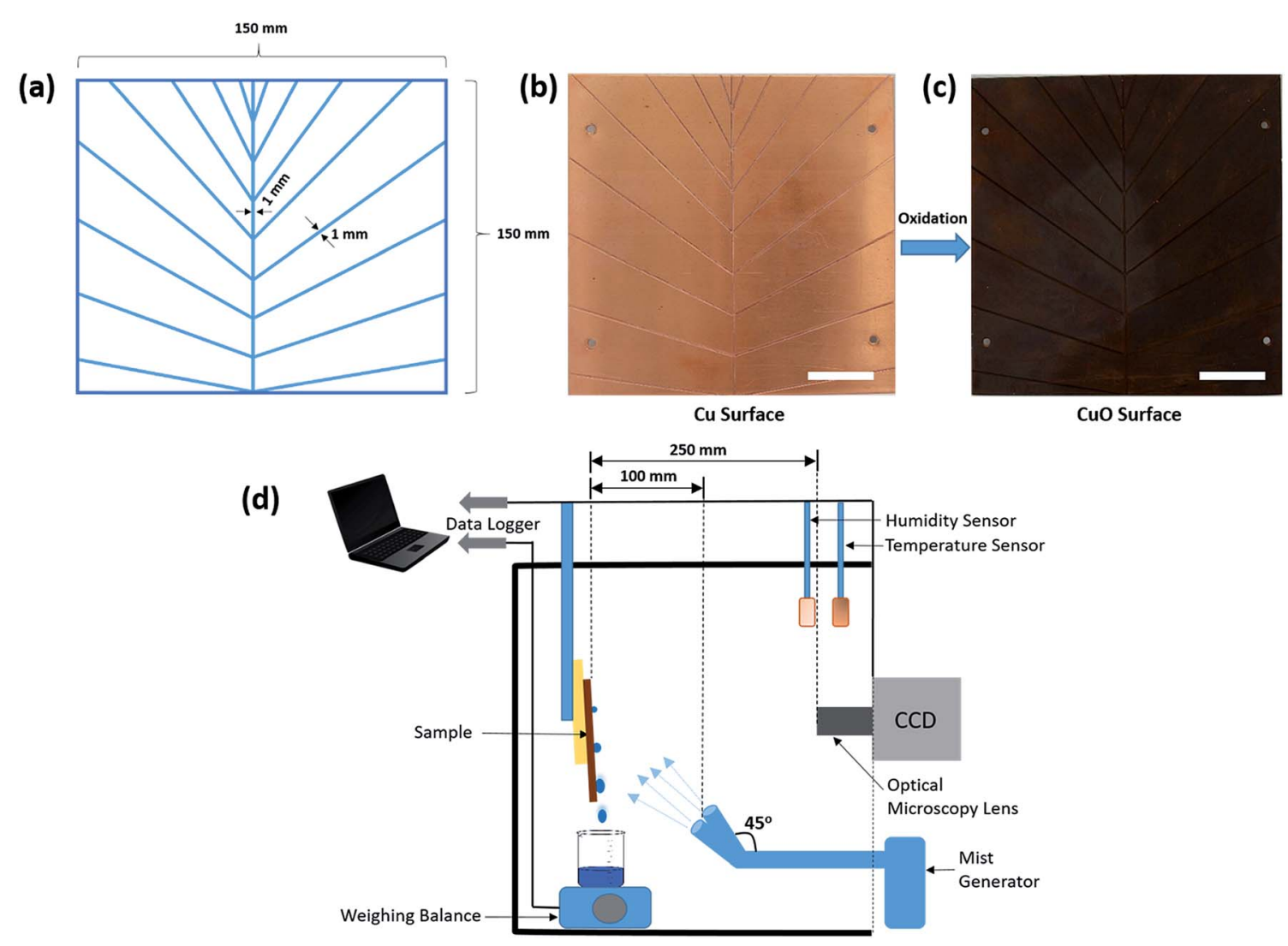

Fig. 1 (a) Picture showing the design, dimensions and channel orientation. (b) and (c) are the unprocessed and processed sample surfaces. The scalebars in (b) and (c) are $30 \mathrm{~mm}$. (d) Schematic of the fog harvesting setup for water collection. 
monochromatized $\mathrm{Mg} \mathrm{K} \alpha$ X-rays (1253.6 eV) generated using a DAR400 X-ray source (Omicron Nanotechnology GmbH) operated at $300 \mathrm{~W}$ for excitation of photoelectrons and Auger electrons. The measurements were carried out in normal emission with a detection area of $2.93 \mathrm{~mm}^{2}$ (Ø1.93 mm). The core level spectra were collected with a pass energy of $20 \mathrm{eV}$ employing an Argus hemispherical electron spectrometer (Omicron Nanotechnology $\mathrm{GmbH}$ ) installed in a multifunctional ultra-high vacuum (UHV) surface analysis system. ${ }^{\mathbf{5 4}}$ The surface compositions were identified by analyzing core level spectra using Casa XPS software (Version 2.3.18 PR 1.0). The binding energy scale was calibrated according to a metallic Ag $3 \mathrm{~d}_{5 / 2}$ reference at $368.00 \mathrm{eV}$. The background subtracted XPS peaks were least-squares fitted with a combination of symmetric Gaussian-Lorentzian component line shapes, while the $\mathrm{Cu}$ LMM transition was fitted using experimentally obtained line shapes of $\mathrm{Cu}^{0}, \mathrm{Cu}^{+}$, and $\mathrm{Cu}^{2+}$ references. ${ }^{55}$ The relative atomic concentrations were calculated using Scofield photoionization cross-sections and an experimentally measured transmission function of the Argus analyzer. ${ }^{56}$

High-resolution surface structure imaging characterization was performed using a FESEM (field emission scanning electron microscope) (Zeiss UltraPlus) operating at $15 \mathrm{kV}$. Elemental analysis was done using an EDS (Energy dispersive X-ray spectroscopy) attachment linked to FESEM (Oxford Instruments XMaxN 80 EDS). Contact angle measurements were done by using a video-based optical contact angle system (Theta Lite Optical Tensiometer, Biolin Scientific). Three independent sessile drop measurements were performed using OneAttension software and values were extracted via the tangent method.

\section{Fog collection experiments}

The experimental setup for the determination of the fogcollection rate is shown in Fig. 1d. Fog collection experiments were done by exposure of the surfaces to the fine mist of ultrapure water generated by two commercial cool mist ultrasonic humidifiers (Bionaire BU1300W-I) working at the same time and having one output nozzle each. The nozzles were tilted to an angle of $45^{\circ}$ from the vertical position as shown in Fig. 1d to make sure that the fog flow reaching the surface is not too weak and covers the whole surface. ${ }^{38,57}$ In the literature, it has been reported that the typical wind speed of the fog in a desert is around $10-50 \mathrm{~mm} \mathrm{~s}^{-1} .^{58}$ In order to obtain similar conditions and a homogeneous fog distribution across the surface, the humidifiers which emit the cool mist in the range of 50-150 mm $\mathrm{s}^{-1}$ (measured using a Jessiekervin YY3 hand-split digital anemometer) were placed at a distance of about $100 \mathrm{~mm}$ from the sample mounted vertically to the stage. The samples were slightly tilted from the vertical orientation during the experiments to aid the droplet movement on channels. This is to avoid the droplets overshooting the channels which may foul the other nucleating areas and thus prematurely ending the growth of other droplets. The aerodynamic conditions may vary slightly due to minor variation in the output from the humidifier. This suggests the use of a tilted surface with respect to the direction of the wind for better fog collection efficiency. However, the results are reliable and a comparative study is possible if the sample size and the position are same, hence experimental conditions were kept the same every time with special care. The total air flow for both the humidifiers was estimated as ca. $260 \pm$ $60 \mathrm{~mL} \mathrm{~h}{ }^{-1}$. The experimental setup had a relative humidity above $90 \%$ and the temperature was maintained at $24{ }^{\circ} \mathrm{C}$ throughout the experiments. The size of the droplets in this case typically ranges from 1 to $5 \mu \mathrm{m}$ in diameter, ${ }^{59}$ which is similar to those reported elsewhere ${ }^{38}$ and comparable to that of atmospheric fog. Then, the volume of water was collected and measured with a weight balance at intervals of 20 min over a period of $2 \mathrm{~h}$. For the onset of dripping experiments, the surfaces used were $15 \mathrm{~mm} \times 15 \mathrm{~mm}$ in dimensions and were freshly and independently fabricated. The irregularities on the
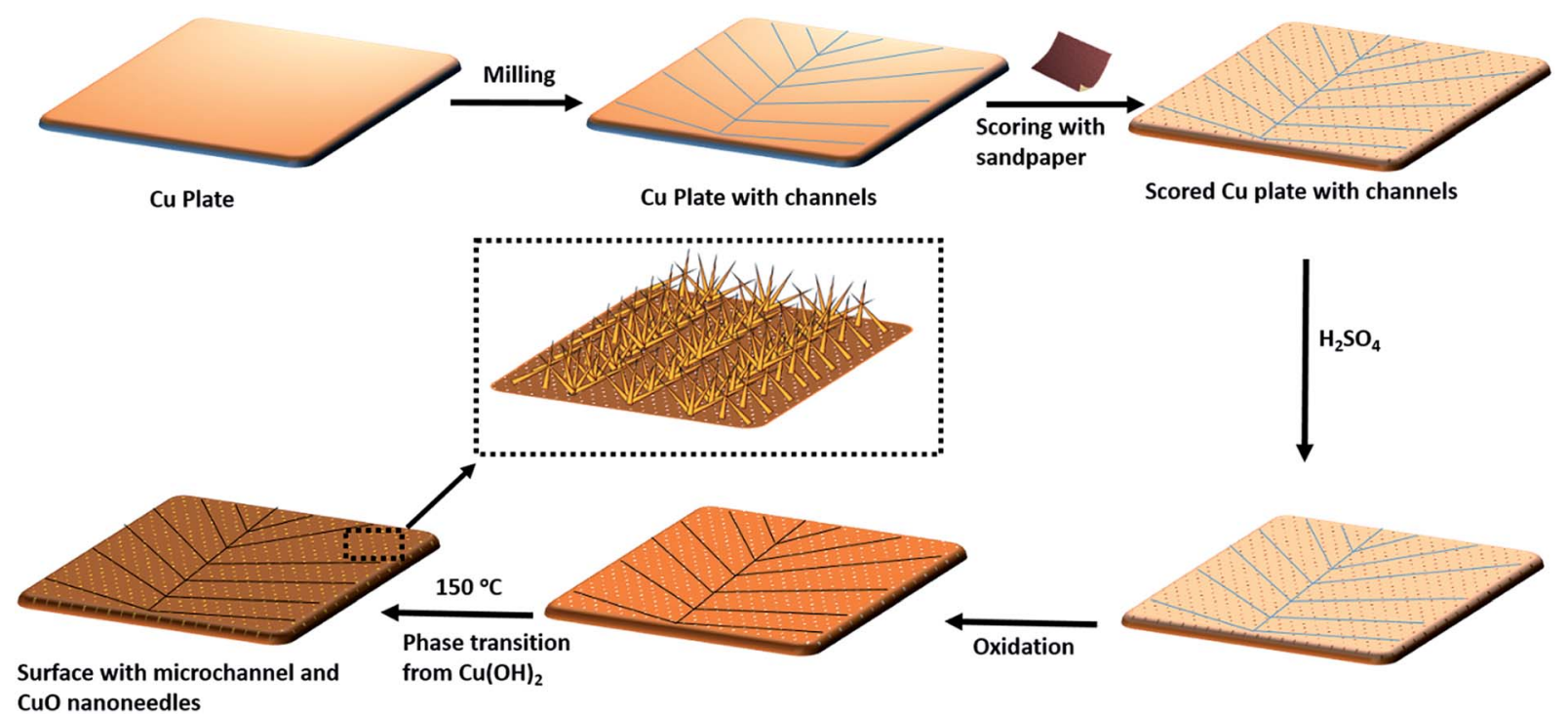

Fig. 2 Schematic of large-scale fabrication of patterned high-density $\mathrm{CuO}$ nanoneedle structures. 
edge of the surfaces may influence the volume of the falling droplets. ${ }^{60}$ As the droplet deposition on to the surface is dependent on the wettability of the surface and the surfaces were independently fabricated after cutting, it can be reasonably assumed that edge effects are negligible and can be ignored. Three sets of trials were executed on three independent samples and the average values along with the standard deviation were calculated. Special care was taken to ensure the same experimental conditions for all measurement rounds.

\section{Results and discussion}

Fig. 2 shows the schematic of the sketch of the fabrication of patterned high-density $\mathrm{CuO}$ nanoneedle structures. In this study, a large scale, low cost and facile oxidation process was introduced. To characterize the surface morphology of the fabricated CuO surfaces, FESEM was employed and the images at different scales along with their corresponding EDS spectra are shown in Fig. 3. Fig. 3a1 and a2 show the original $\mathrm{Cu}$ surface which has a relatively planar structure and the EDS spectra in Fig. $3 \mathrm{c} 1$ confirms the presence of only copper. The surface roughness consisting of the directional protrusions was induced on to the surfaces of smooth $\mathrm{Cu}$ with the simple scoring using sandpaper which can be seen in Fig. $3 \mathrm{~b}$. The oxidation process led to the formation of the uniform high- density $\mathrm{CuO}$ nanoneedles with a high aspect ratio which is evident in Fig. 3c. The corresponding EDS spectra in Fig. 3c3 confirm the existence of copper as copper oxide as the presence of oxygen is clearly evident in addition to copper. From Fig. 3c, it can be seen that the typical length of these nanoneedles is in the range of a few $\mu \mathrm{m}$ and the needles are high aspect ratio conical structures. Some micro-flower like structures composed of $\mathrm{CuO}$ can also be observed from Fig. 3c, which may be the result of $\mathrm{Cu}$ etched from the surface.

The nanoneedles were conceivably composed of hydrated copper oxide which usually forms during the oxidation process of copper. ${ }^{61}$ The oxidation process usually proceeds in three steps. In the first step, the growth of $\mathrm{Cu}(\mathrm{OH})_{2}$ occurs which can be explained by the existence of $\mathrm{NaOH}$ and $\left(\mathrm{NH}_{4}\right)_{2} \mathrm{~S}_{2} \mathrm{O}_{8}$ which allows rapid oxidation of $\mathrm{Cu} .{ }^{62} \mathrm{NaOH}$ alone may also oxidize the surface, but it has been reported that it might take a longer time. ${ }^{63} \mathrm{Cu}^{2+}$ cations released from this process react with the hydroxide anions to form $\mathrm{Cu}(\mathrm{OH})_{2}$ on the surface. During this process, gas bubbles with a characteristic ammonia odor were noticed which indicated the formation of $\mathrm{NH}_{3}$. This step can be summarized as an equation: ${ }^{\mathbf{6 4}}$

$$
\begin{aligned}
& \mathrm{Cu}(\mathrm{s})+4 \mathrm{NaOH}(\mathrm{aq})+\left(\mathrm{NH}_{4}\right)_{2} \mathrm{~S}_{2} \mathrm{O}_{8}(\mathrm{aq}) \rightarrow \\
& \mathrm{Cu}(\mathrm{OH})_{2}(\mathrm{~s})+2 \mathrm{Na}_{2} \mathrm{SO}_{4}(\mathrm{aq})+2 \mathrm{NH}_{3}(\mathrm{~g})+2 \mathrm{H}_{2} \mathrm{O}
\end{aligned}
$$
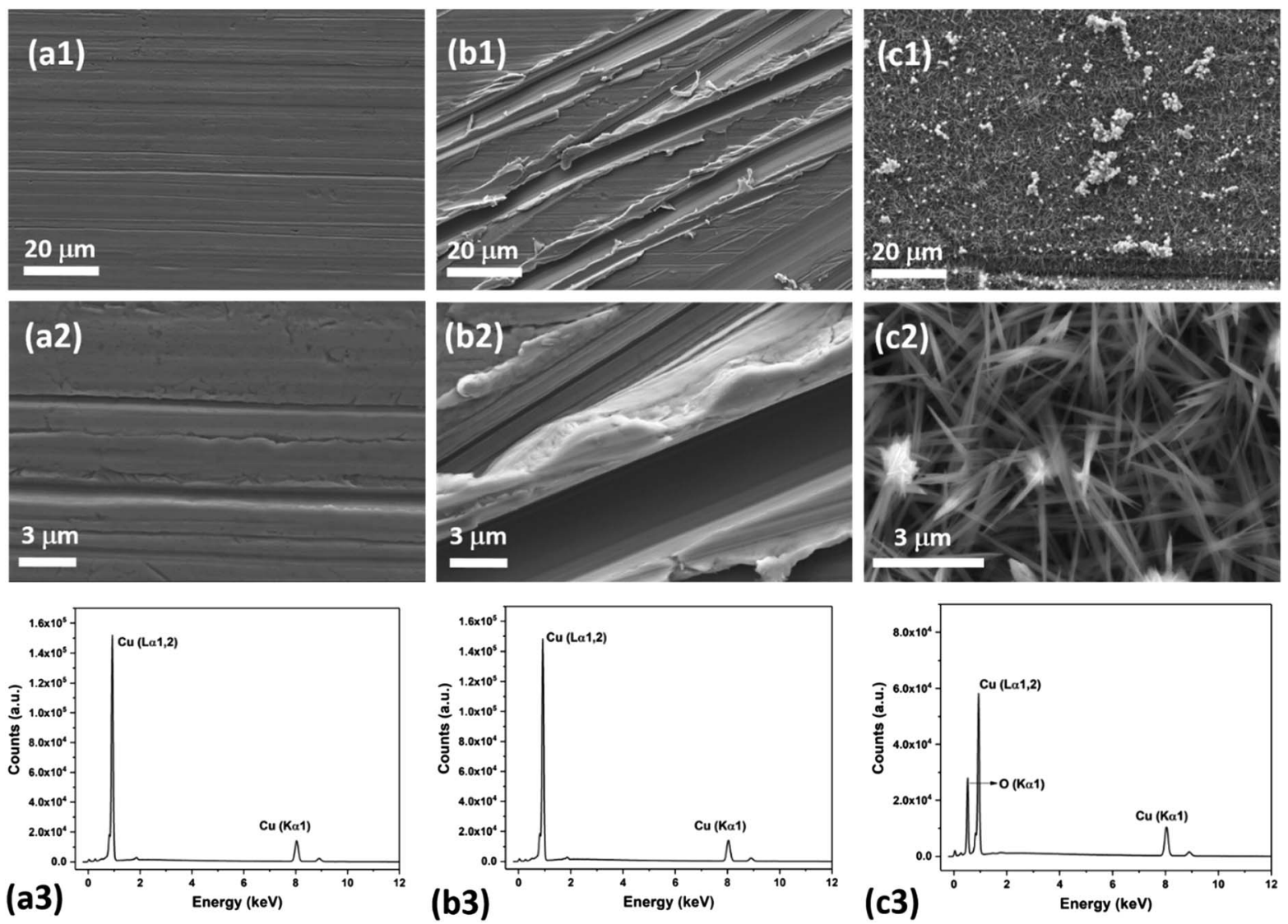

Fig. 3 SEM images of the uncoated bare $\mathrm{Cu}(\mathrm{a})$, uncoated scored $\mathrm{Cu}(\mathrm{b})$ and uncoated $\mathrm{CuO}$ nanoneedle surfaces (c) at different resolutions (1 and 2). (3) Corresponding EDS spectra of the surfaces. The CuO nanoneedle samples here were 2 days old and kept under ambient conditions. 
In the second step $\mathrm{Cu}(\mathrm{OH})_{2}$ transforms very rapidly into tetrahydroxocuprate (II) anions $\mathrm{Cu}(\mathrm{OH})_{4}{ }^{2-}$ due to the alkalinity of the solution (eqn (2)). ${ }^{65}$ In the final step, $\mathrm{Cu}(\mathrm{OH})_{4}{ }^{2-}$ prepared in the previous step can be considered as the precursor for the growth of $\mathrm{CuO}$ nanoneedles which are formed by the reaction shown in eqn (3).

$$
\begin{gathered}
\mathrm{Cu}(\mathrm{OH})_{2}(\mathrm{~s})+2 \mathrm{OH}^{-}(\mathrm{aq}) \rightarrow \mathrm{Cu}(\mathrm{OH})_{4}{ }^{2-} \text { (aq) } \\
\mathrm{Cu}(\mathrm{OH})_{4}{ }^{2-}(\mathrm{aq}) \leftrightarrow \mathrm{CuO}(\mathrm{s})+2 \mathrm{OH}^{-}(\mathrm{aq})+\mathrm{H}_{2} \mathrm{O}
\end{gathered}
$$

The small CuO particles prepared in this step have high surface energy and are highly reactive, so these aggregate around the 3D protrusions created by scoring and form the nanoneedle-like structures. In the absence of scoring with sandpaper, nanostructures in a random shape and orientation could be formed which may lead to non-wetting surfaces (refer Fig. S1a, ESI $\dagger) .{ }^{63}$ It has been reported that by optimizing the etching time and reactants in similar oxidation approaches, a variety of surface morphologies could be achieved. ${ }^{63,64,66}$ In this case, the presence of $\mathrm{H}_{2} \mathrm{SO}_{4}$ played a crucial role in the formation of nanoneedle-like structures which were uniformly spread over a large area and the same structures were obtained every time in repeated fabrication experiments. The formation of such structures may be due to the blocking of $\mathrm{Cu}$ layers by bisulfate ions. A number of studies on the interaction of sulfuric acid species with metallic surfaces are reported in the literature.$^{67}$ A comparative study on the adsorption of sulfuric acid species on $\mathrm{Cu}$ surfaces along with other surfaces revealed that bisulfate adsorbs very effectively on to the surface of $\mathrm{Cu} .{ }^{67}$ Thus, when the oxidation process takes place, bisulfate ions strongly adsorb on the freshly formed surface of $\mathrm{CuO}$ and prevent $\mathrm{CuO}$ particle deposition throughout the surface which leads to the formation of high density $\mathrm{CuO}$ nanoneedles on the surface. This can be confirmed with the SEM image of the $\mathrm{CuO}$ surfaces (Fig. S1b, ESI $\dagger$ ) fabricated in the absence of $\mathrm{H}_{2} \mathrm{SO}_{4}$ which displayed nanoflake-like structures.

The surface chemistry of the $\mathrm{CuO}$ nanoneedles and their surface modification with hydrophobic coating were investigated by XPS which is shown in Fig. 4. According to XPS, the bare $\mathrm{Cu}$ surface contained $\mathrm{C}-\mathrm{C} / \mathrm{H} / \mathrm{O}$ species (C: 83.8 at\%) and some $\mathrm{Cu}^{+}$oxide on top of the metallic $\mathrm{Cu}$. As seen in Fig. $4 \mathrm{~b}$, the $\mathrm{Cu}$ LMM peaks on the bare $\mathrm{Cu}$ at $917.1 \mathrm{eV}$ and $919.0 \mathrm{eV}$ (Auger parameters: $1849.4 \mathrm{eV}$ and $1851.3 \mathrm{eV}$ ) correspond to $\mathrm{Cu}^{+}$and $\mathrm{Cu}^{0}$, respectively. The components of $\mathrm{O} 1 \mathrm{~s}$ at $530.2 \mathrm{eV}$ and $\mathrm{Cu}$ $2 \mathrm{p}_{3 / 2}$ at $932.3 \mathrm{eV}$ support the presence of $\mathrm{Cu}^{+}$oxide.

In the case of $\mathrm{CuO}$ nanoneedles, the oxidation process ideally should remove all the carbon species. However, the presence of carbon species on to the surface (C: 26.2 at\%) can be mainly due to the ambient exposure which is known to change the wettability of the CuO based surfaces. ${ }^{33}$ The carbon content in the freshly fabricated surfaces can be lower and depends on the ambient conditions and age of the samples as shown in Fig. S2 (ESI $\dagger$ ). The fabrication process also induced some Si impurity (3.6 at\%) on the surface which can be mainly due to the scoring with sandpaper or the silicon oil used during the milling process. Copper was found only in the oxidation state of
$\mathrm{Cu}^{2+}$ indicated by the $\mathrm{Cu}$ LMM line shape and peak position at 918.1 eV (Auger parameter: $1851.8 \mathrm{eV}$ ) which confirmed that the nanoneedles are composed of $\mathrm{CuO}$. The components of $\mathrm{O} 1 \mathrm{~s}$ at $529.5 \mathrm{eV}$ and $\mathrm{Cu} 2 \mathrm{p}_{3 / 2}$ at $933.7 \mathrm{eV}$ with a strong shakeup satellite support the presence of $\mathrm{Cu}^{2+}$ oxide. $\mathrm{Si}^{2+}$ (2.9 at\%), $\mathrm{Si}^{4+}(0.4 \mathrm{at} \%)$, and carbon-bonded $\mathrm{F}$ (2.1 at\%) were detected on the $\mathrm{CuO}$ nanoneedle surface after silanization (Fig. 4c). Copper remained in the oxidation state of $\mathrm{Cu}^{2+}$ as indicated by the $\mathrm{Cu}$ LMM line shape and peak position at $918.1 \mathrm{eV}$ (Auger parameter: $1851.6 \mathrm{eV}$ ). The components of $\mathrm{O} 1 \mathrm{~s}$ at $529.4 \mathrm{eV}$ and $\mathrm{Cu} 2 \mathrm{p}_{3 /}$ ${ }_{2}$ at $933.5 \mathrm{eV}$ with a strong shakeup satellite support the presence of $\mathrm{Cu}^{2+}$ oxide. Bisulfate adsorption on to the surface of $\mathrm{Cu}$ was observed before the oxidation process which further strengthens our point that the presence of $\mathrm{H}_{2} \mathrm{SO}_{4}$ is necessary for the $\mathrm{CuO}$ nanoneedle growth process (refer Fig. S3, ESI $\dagger$ ). The results confirmed that the surfaces obtained after the oxidation process were predominantly composed of $\mathrm{CuO}$ and rule out the presence of any other $\mathrm{Cu}$ oxide species.

\section{Wettability studies}

The ability of the surface to capture water depends on the wettability of the surface, so we characterized the wetting properties of the surfaces in detail. The wetting properties of the $\mathrm{CuO}$ nanoneedles were studied using droplet spreading experiments and contact angle measurements. The sequence of captured images during droplet spreading of a $1 \mu \mathrm{L}$ water droplet on the uncoated surfaces can be seen in Fig. 5a. As soon as the droplet is dispensed on to the $\mathrm{CuO}$ nanoneedle surface without any coating after fabrication, the wetting of the water droplet proceeds in two phases: in the first phase, it wets the surface very quickly and reaches a diameter of $\sim 3-4 \mathrm{~mm}$ in less than $0.1 \mathrm{~s}$. In the second phase, the drop continues to spread slowly, and reaches a diameter of $\sim 6-7 \mathrm{~mm}$ in $20 \mathrm{~s}$, displaying very low contact angles. Immediately after the drop was dispensed on to the surface, a precursor perimeter was observed which was evidenced to be outside the periphery of the water contact line. During the second, slow phase of the wetting, the droplet spreading continued linearly with time despite the evaporation (refer Video S1, ESI $\dagger$ ) till it reaches its maximum diameter.

The water-spreading was also investigated on the control samples. In the case of bare $\mathrm{Cu}$ surface (Fig. S4a $\dagger$ ) the water droplet that touched the surface did not spread out and maintained a contact line of about $4 \mathrm{~mm}$ over time. A similar wetting behavior was observed in the case of scored $\mathrm{Cu}$ surface (Fig. S4b $\dagger$ ) where the droplet also maintained a constant contact line, however, the shape of the droplet sitting on the surface was more spherical in appearance. This hence confirmed the role of the high-density distribution of nanoneedles in efficient spreading of the water droplets. The waterspreading under capillary and viscous frictional effects of the $\mathrm{CuO}$ nanoneedle surfaces are shown via the schematic in Fig. 5b. After immediate dispensing of the water droplet on the surface, some quantity of water quickly spreads to the base via the tip along the surfaces of nanoneedles. It has been well established that the conical microstructures are responsible for 

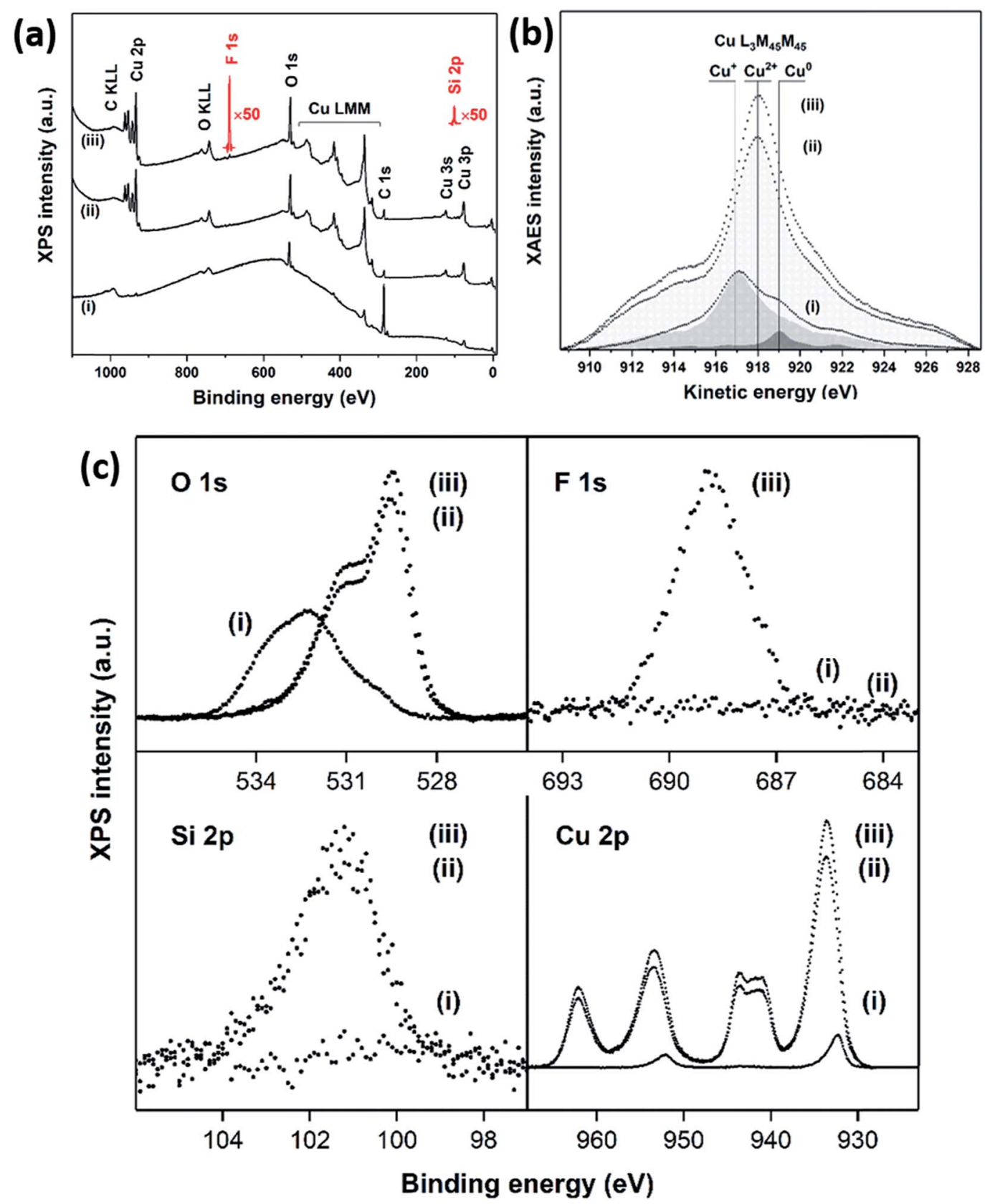

Fig. 4 (a) XPS survey spectra (black) and selected low-intensity core level spectra which are not clearly visible in the survey scan (red). (i) Bare Cu, (ii) $\mathrm{CuO}$ nanoneedles and (iii) silanized $\mathrm{CuO}$ nanoneedles. (b) XAES Cu $\mathrm{L}_{3} \mathrm{M}_{45} \mathrm{M}_{45}$ spectra of (i) bare $\mathrm{Cu}_{1} \mathrm{Cu}^{0}: 10.9 \%, \mathrm{Cu}^{+}$: $89.1 \%$, (ii) $\mathrm{CuO}$ nanoneedles, $\mathrm{Cu}^{2+}: 100 \%$ and (iii) silanized $\mathrm{CuO}$ nanoneedles, $\mathrm{Cu}^{2+}: 100 \%$. Measured data are shown as dots. Fitted experimental reference line shapes in the greyscale fill are shown for bare Cu surface and oxidized surfaces. (c) High-resolution XPS spectra of O 1s, F 1s, Si 2p, and Cu 2p transitions. The exposure time for uncoated $\mathrm{CuO}$ nanoneedles used in XPS analysis was 7 days from the fabrication.

the increase in the pressure gradient. ${ }^{19}$ Due to this increase in the pressure gradient, the droplet dispensed on the surface of nanoneedles quickly leads to wetting as soon as it touches the surface. In the next step, a layer of water is formed on its surface which facilitates further quick droplet spreading. It is noteworthy that a complete spreading was not observed on the $\mathrm{CuO}$ nanoneedle surface. Complete spreading can hinder the droplet movement by promoting high adhesion forces. ${ }^{68}$ This is because the droplet spreading on the micro- and the nanostructured surface is related to the balance between the capillary forces and the viscous frictional forces. ${ }^{68,69}$ As the size of the microstructures is decreased beyond a certain level, the viscous frictional forces start to dominate the capillary forces. Since we observed efficient droplet spreading without fully wetting the whole $\mathrm{CuO}$ nanoneedle surface, it means that the nanoneedles had a suitable feature size and inter-texture distances which maintained a balance between the capillary force and the viscous frictional force. It is also noteworthy that the wettability of the $\mathrm{CuO}$ 
(a)
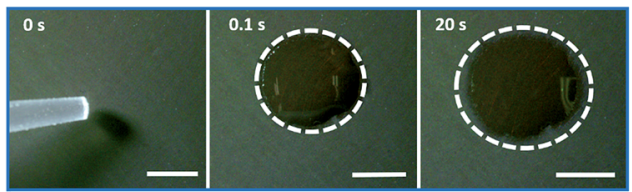

(b)

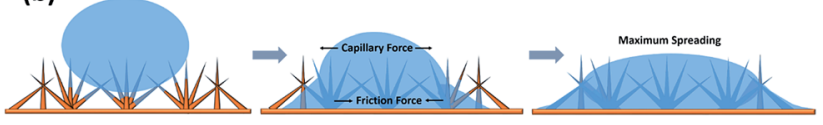

Fig. 5 (a) Images of a water droplet $(1 \mu \mathrm{L})$ spreading on hydrophilic $\mathrm{CuO}$ nanoneedle surface and (b) schematic diagram of the spreading of a water droplet. The scale bar in (a) is $2 \mathrm{~mm}$.

nanoneedle surface is subject to the structure-coupled adsorption of airborne volatile organic carbon species, where a transition of the surfaces from superhydrophobic can be observed. ${ }^{33}$ To prove this, we measured the adsorbed carbon content on the surfaces of $\mathrm{CuO}$ using XPS along with change in wettability over the period of 0 to 108 days and the data are shown in Fig. S2, ESI. $\dagger$ It was observed that on ambient exposure of the $\mathrm{CuO}$ surface, the surfaces adsorb volatile organic carbon which changes its wettability from superhydrophilic to superhydrophobic.

In the case of all surfaces with hydrophobic coating, no change in contact radius or droplet morphology was observed and the droplets maintained a spherical shape throughout the experiment. However, it is expected that the contact radius on the hydrophobic surfaces steadily decreases over time due to the evaporation of the droplet. ${ }^{70}$ In our studies, we noticed that the $\mathrm{CuO}$ nanoneedle surface with hydrophobic coating displayed a very high contact angle $\left(>165^{\circ}\right)$ and a low contact angle hysteresis $\left(<10^{\circ}\right)$. Usually, the Cassie state is compromised when small droplets are present on the surface and often the pinning effect is seen. However, in this case, it was observed that even with the small droplet size (Fig. S5, ESI $\dagger$ ), the stability of the Cassie state could be maintained. This can be attributed to the aspect ratio and density of the nanoneedles, i.e. even the smallest observable drops are much larger than the feature size of the surface. Control samples with silane coating were also observed to be hydrophobic, but with a very high contact angle hysteresis (Fig. 6). Droplet pinning was observed in the case of both the hydrophobic control samples, which may be due to the irregularities in the surface morphology and lack of periodic microstructures. Thus, the unique interaction of water droplets with surfaces composed of uncoated and coated $\mathrm{CuO}$ nanoneedles makes them a suitable candidate for the enhancement in droplet nucleation, growth and efficient removal of deposited water. The fabrication of these surfaces can be easily scaled up with ease and is ideal for the development of materials and practical devices that can harvest the atmospheric water with high efficiencies.

\section{Fog harvesting studies}

The hierarchical topography of the $\mathrm{CuO}$ nanoneedle surfaces discussed in this paper has two major key phases in enhancing the atmospheric water harvesting efficiency. First is the rapid nucleation, growth and departure/shedding of the water droplets on the hierarchical conical structures inspired by Opuntia microdasys ${ }^{19}$ and Cynodon dactylon. ${ }^{17}$ The second aspect is the guided transport of the water droplets for the efficient collection without fouling the other areas where nucleation and growth of the water droplet are happening.

The fog collection efficiencies of the $\mathrm{CuO}$ nanoneedle samples along with controls were determined in three measurement cycles and the water-harvesting test was performed as described in the Materials and methods section. Samples with some of the processing steps omitted were used as the control samples and their contact angles are shown in Fig. 6. The characteristic snapshots of fog harvesting experiments on different samples at different time intervals can be found in Fig. 7. Upon impact of the tiny fog droplets with the surface, first droplets are visible on the surface by heterogeneous coalescence. As the fog harvesting experiment proceeds, droplets suspended in the fog flow reaching the surface now coalesce with already collected droplets. After a few seconds, droplets with sizes ranging from hundreds of $\mu \mathrm{m}$ to few $\mathrm{mm}$ can be observed in the case of hydrophilic $\mathrm{CuO}$ nanoneedle surfaces and $\mathrm{CuO}$ nanoneedle surface with hydrophobic coating. It can
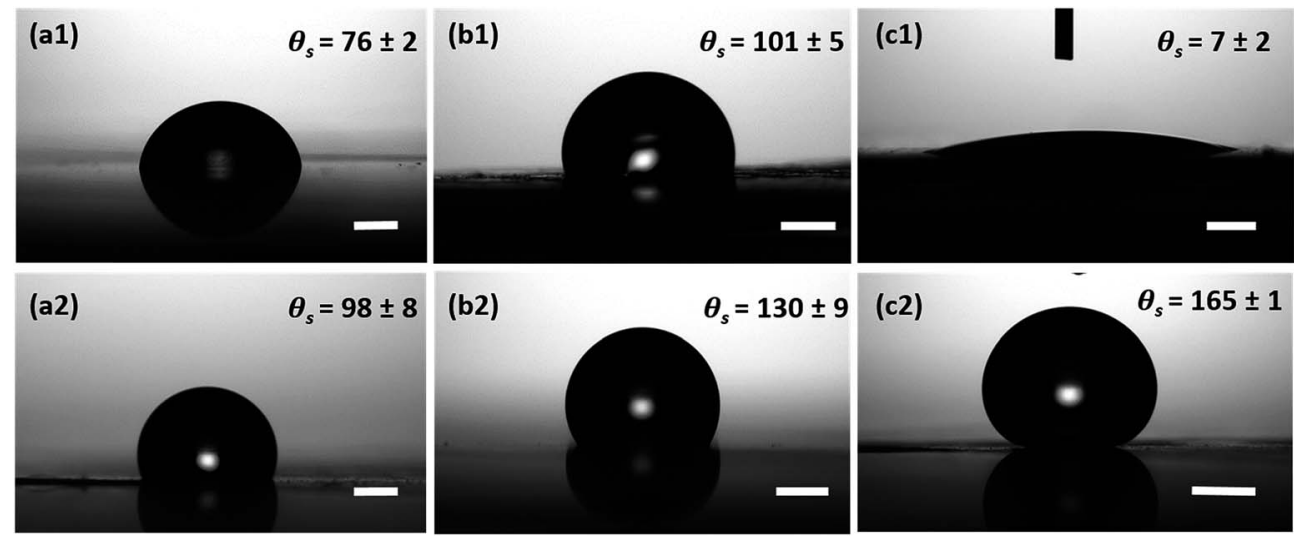

Fig. 6 Contact angle $\theta$ (deg) images of (1) uncoated and (2) hydrophobic coated surfaces composed of (a) bare Cu surface, (b) scored Cu surface and (c) $\mathrm{CuO}$ nanoneedle surfaces. The scale bars in the images are $1 \mathrm{~mm}$ and the volume of water is $\sim 6 \mu \mathrm{L}$. 

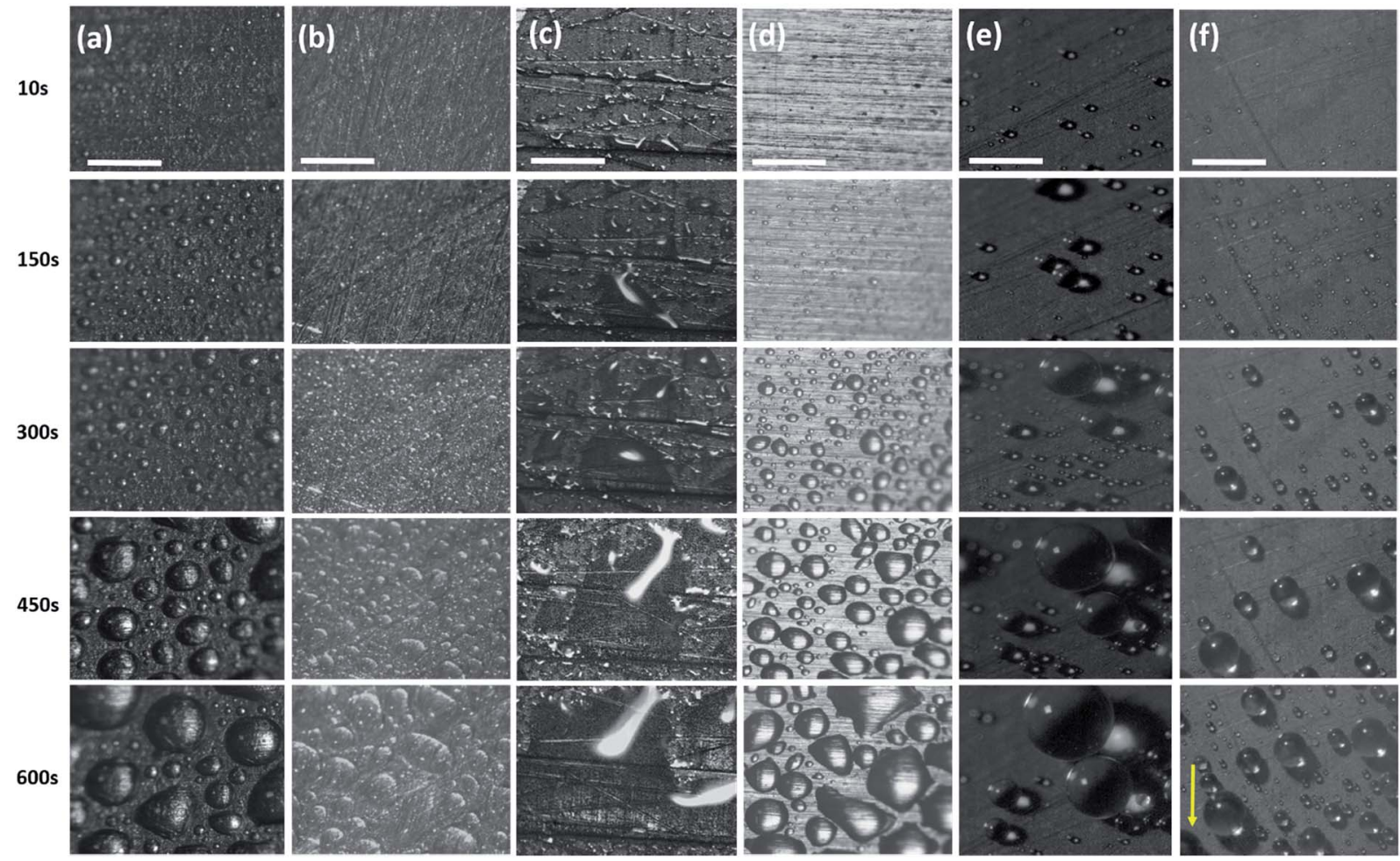

Fig. 7 Snapshots of droplet formation and transport on different surfaces: (a) uncoated bare $\mathrm{Cu}$, (b) uncoated scored $\mathrm{Cu}$, (c) uncoated $\mathrm{CuO}$ nanoneedles, (d) coated bare $\mathrm{Cu}$, (e) coated scored Cu and (f) coated CuO nanoneedles at time intervals 0, 150, 300, 450 and 600 s. Scale bars are $1 \mathrm{~mm}$.

be evidenced that comparatively fewer droplets were observed in all the other control surfaces in the initial phase. The shapes of a droplet in the surfaces put in the inclined position are predicted by contact angle hysteresis (a measure of advancing and receding contact angles) as well as the types of their corresponding nanostructures. ${ }^{71}$ In the initial phase of the experiment, the droplets appear to be spherical in shape in all the samples. Eventually, as the droplets grow with the size similar to the capillary length $\left(l_{\mathrm{c}}=[\gamma /(\rho g)]^{0.5}\right.$, where $\gamma$ is the surface tension between the liquid and gas, $\rho$ is the density of water and $g$ denotes gravity), the factor of gravity comes into play and the shape of the droplets starts to deform. ${ }^{72}$ It can be seen in Fig. 6 that the droplets in the case of all the non-hydrophobic samples deviate from the perfect spherical cap figure. It is noteworthy that the shape of the droplets shows a different orientation on the hydrophilic $\mathrm{CuO}$ nanoneedle surface than the nonhydrophobic control samples. In the case of the hydrophilic $\mathrm{CuO}$ nanoneedle surface, most droplets orient slightly in the vertical direction under the effect of gravity, while on the control samples without the nanoneedles, most droplets stretch in the random direction and seem to make a film on the surface. The different orientation of the droplets on the hydrophilic $\mathrm{CuO}$ nanoneedle surface can be attributed to the arrangement of the nanostructures and droplet spreading aided by the gravitational force. The different droplet morphologies observed in the current experiments require further investigations at large scale as well as longer time durations to be able to evaluate the impact of the action of vertical and horizontal forces on the drops and whether droplet spreading via capillary force or any other forces has any connection with this. In the case of surfaces bearing hydrophobic coatings, the spherical shapes could be observed throughout the experiments. However, in the case of CuO nanoneedle surface with hydrophobic coating, the droplets were comparatively smaller and started rolling off after they reached a certain size. In control samples, the droplets grow bigger in size and remain pinned to the surface for a prolonged period of time which can be due to the instabilities in their Cassie states.

The water removal performance on the different surfaces as the amount of water collected is directly related to the different forces acting on the droplet and to the droplet adhesion to the surface. To quantify the water collection performance on the $\mathrm{CuO}$ nanoneedle-based surfaces as well as the control surfaces, the amount of water dripping from the surfaces was quantified every $20 \mathrm{~min}$ and the data are presented in Fig. 8a. The mass of the first droplet to roll and fall off along with droplet formation time was also compared along with the frequency of the falling droplets (Fig. S6, ESI†). The volume and onset time of the first single falling droplet can be a measure of the fog harvesting ability and water removal quality of the surfaces. ${ }^{73}$ The surfaces displaying short onset time and large droplet size can be considered as more suitable for application in fog harvesting. Throughout the $2 \mathrm{~h}$ of the fog collection experiments, the surfaces exhibiting the nanoneedles without and with hydrophobic coating collected $27.0 \pm 1.4$ and $29.5 \pm 1.5 \mathrm{~mL}$ of water respectively. For a sample size of $22500 \mathrm{~mm}^{2}$ which was 

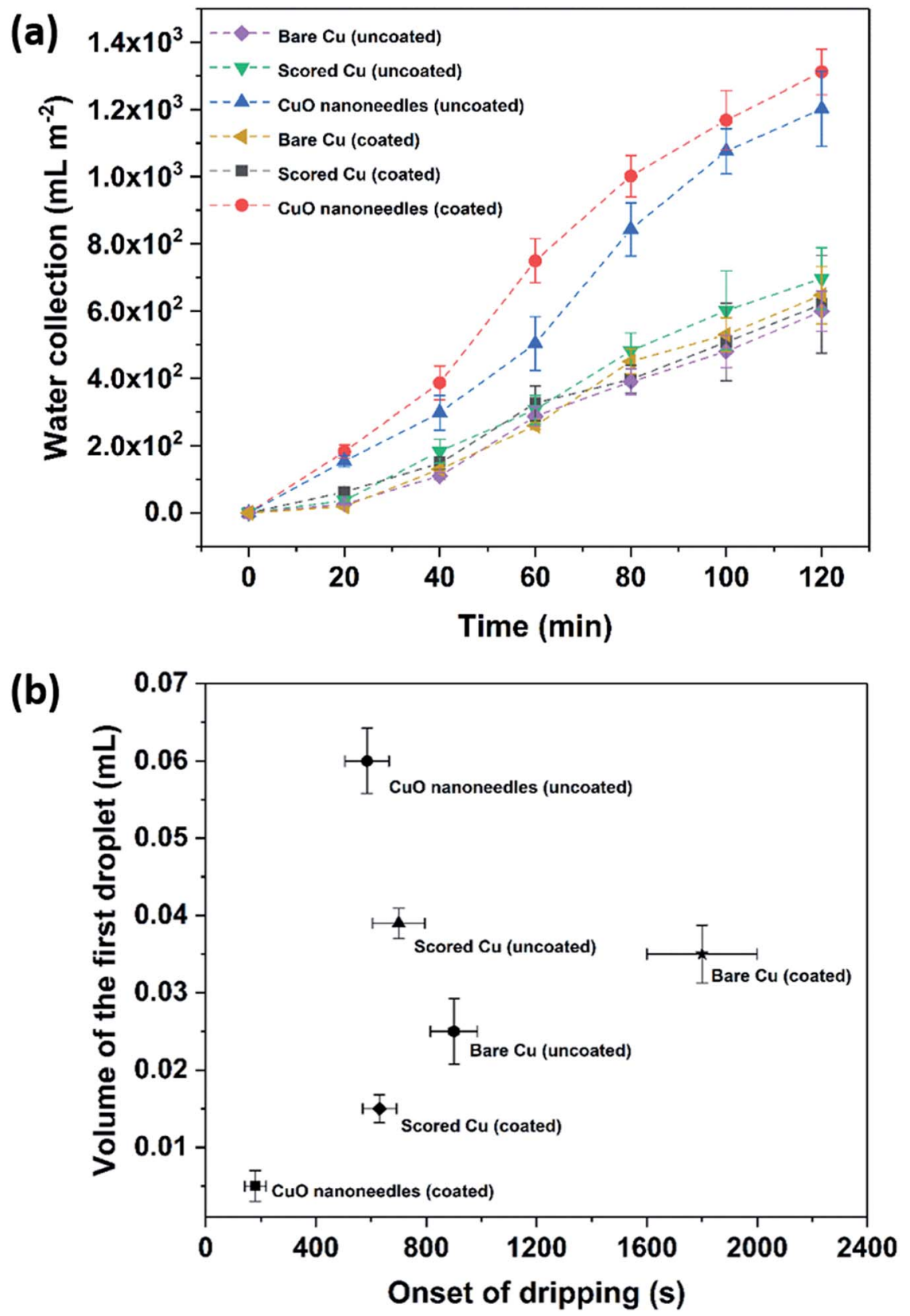

Fig. 8 (a) Fog harvesting dynamics as volume of water collected per square meter, $V\left(\mathrm{~mL} \mathrm{~m}^{-2}\right)$, in time, $t$ (min), over a surface area of $22500 \mathrm{~mm}$ for all surfaces. (b) Average volume and onset time of the first single droplet detaching from the surfaces.

considered in our experiments, the overall amount of water collected per square meter per hour was $600 \pm 30 \mathrm{~mL} \mathrm{~m}^{-2} \mathrm{~h}^{-1}$ for hydrophilic $\mathrm{CuO}$ nanoneedle surface and $655 \pm 35 \mathrm{~mL} \mathrm{~m}^{-2}$ $\mathrm{h}^{-1}$ for $\mathrm{CuO}$ nanoneedle surface with hydrophobic coating. $\mathrm{CuO}$ nanoneedle surface with no hydrophobic coating collected significantly more water as compared to the control surfaces with no hydrophobic coating as large droplets were generated rapidly and began to fall quickly off its surface which is evident from Fig. 8b. The $\mathrm{CuO}$ nanoneedle surface with hydrophobic coating showed the overall best water collection efficiency despite its superhydrophobicity. The superior output of this surface can be attributed to its significantly short onset time (Fig. 8b) and high frequency of falling droplets (refer Fig. S6,
ESI $\dagger$ ), however, the average size of the droplets obtained was not very large. The tendency of these droplets to roll off quickly makes the surface available for the next nucleation and growth cycle which is another interesting trait of these surfaces. It can be evidenced from Fig. 8 that all the control samples yielded a small amount of collected water which can be correlated with their extended onset time. The water collected per square meter per hour was approximately $300 \pm 55,310 \pm 45,325 \pm 45$ and $375 \pm 20 \mathrm{~mL} \mathrm{~m}^{-2} \mathrm{~h}^{-1}$ for bare $\mathrm{Cu}$, scored $\mathrm{Cu}$, hydrophobic coated bare $\mathrm{Cu}$ and hydrophobic coated scored $\mathrm{Cu}$ surfaces, respectively, which is much less than the surfaces displaying the nanoneedle structures. 


\section{Mechanism of fog collection}

The fog harvesting mechanisms on the fabricated surfaces can be explained with the help of the schematics as described in Fig. 9. Clearly, the morphology and the wettability of the surfaces have a direct impact on the water harvesting ability, so the photographs from the wetted surfaces in Fig. 7 and the dynamics of the water collection in Fig. 8 should be considered which make the proposed fog harvesting mechanism more comprehensible. On the uncoated scored and smooth $\mathrm{Cu}$ surface, it starts with the tiny droplet deposition on the surface

(a)

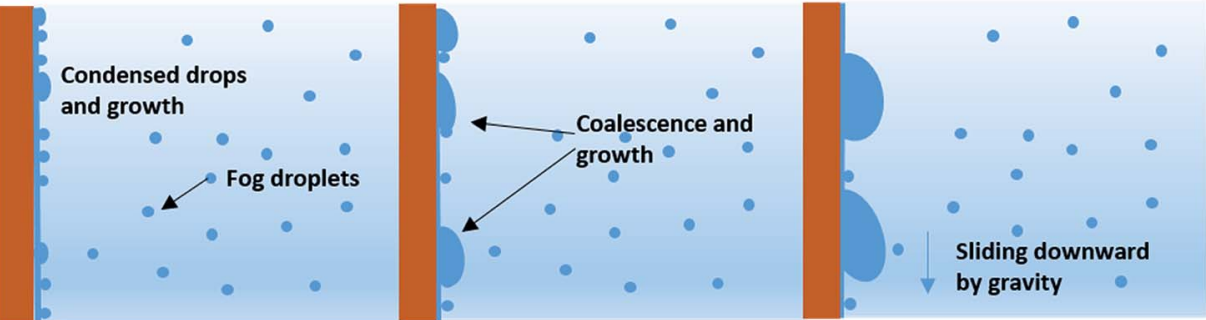

(b)

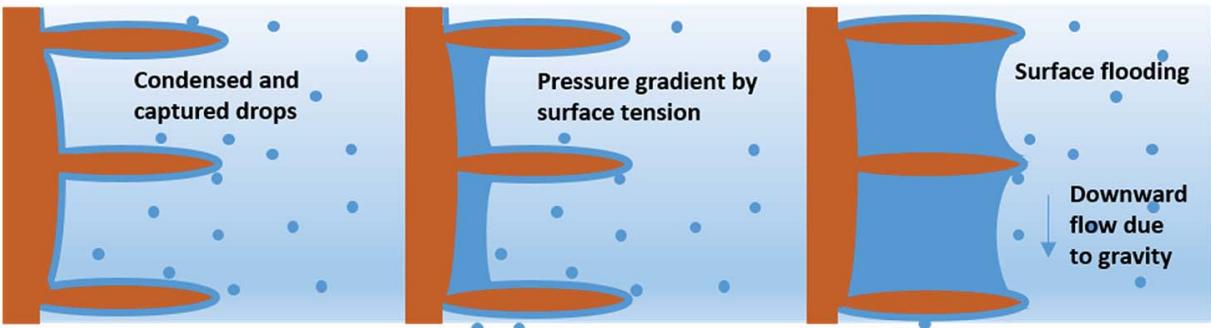

(c)
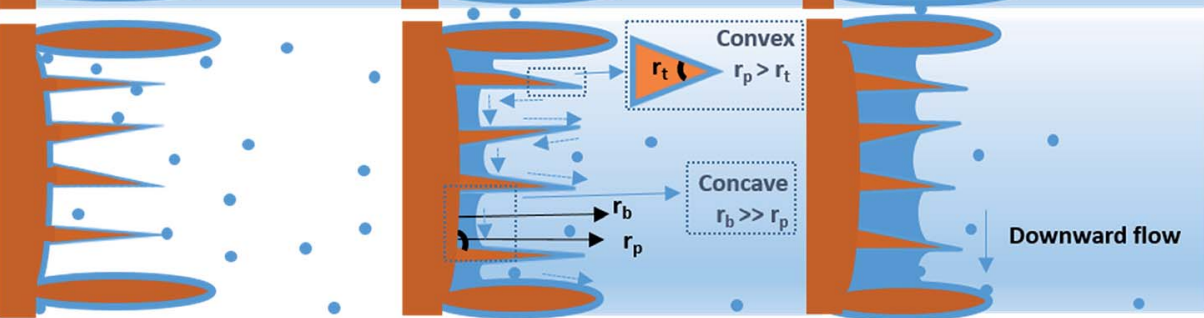

(d)

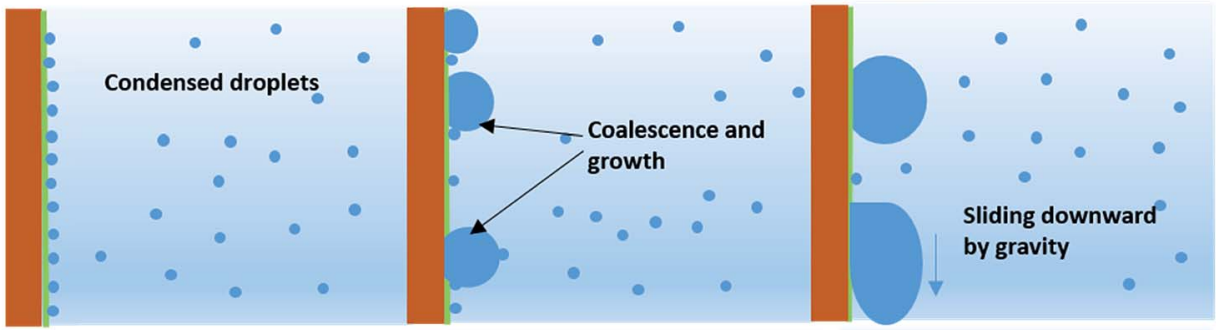

(e)
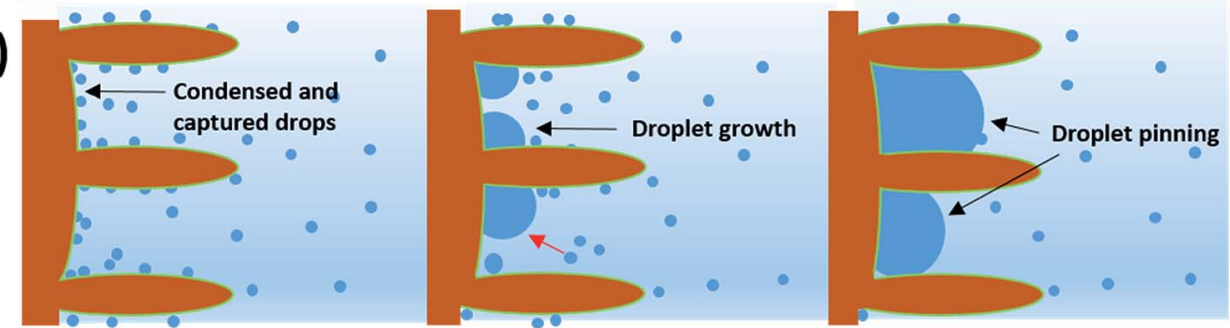

(f)
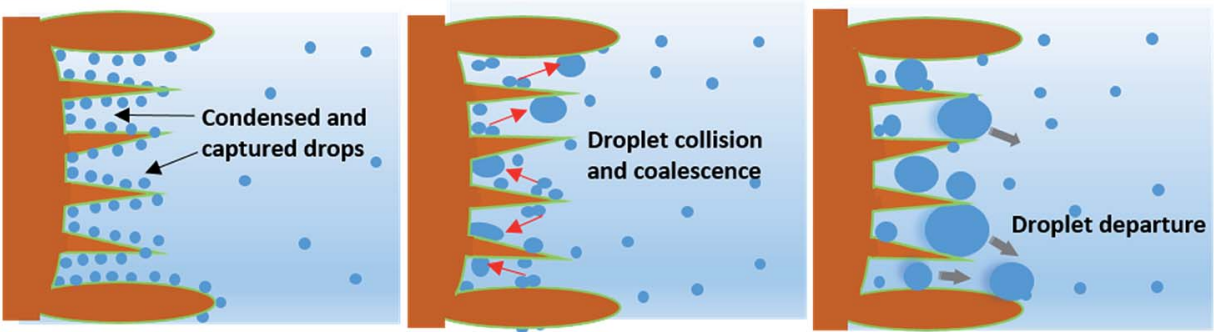

Fig. 9 Schematic graphic showing the fog collection mechanism on (a) uncoated bare $\mathrm{Cu}$, (b) uncoated scored $\mathrm{Cu}$, (c) uncoated CuO nanoneedles, (d) coated bare $\mathrm{Cu}$, (e) coated scored $\mathrm{Cu}$ and (f) coated $\mathrm{CuO}$ nanoneedles. 
which gradually become larger with constant encounter with fog droplets (refer Fig. 7). This is followed by further coalescence of the deposited droplets of random size and shape with the neighboring drops which eventually leads to the formation of the liquid film over the surface. The flow of these water films with the aid of gravity was observed to be very slow, which is responsible for the low water harvesting in these surfaces. On the surfaces bearing nanoneedle structures, a thin film of water was evidenced immediately on the surface at the early stages of the fog exposure which can be attributed to its hydrophilic nature. In this case, the water collection can be defined via pressure gradients on the conical structures. For any microconical structures where the radius of the cones increases from tip to base, a two-dimensional model of the pressure gradient which arises from the surface tension on the film of water is given by: ${ }^{73,74}$

$$
\frac{\mathrm{d} p}{\mathrm{~d} s}=\gamma \frac{\mathrm{d} \kappa}{\mathrm{d} s}
$$

Here, $p$ is the liquid pressure; $s$ is the position of the conical structure and $\kappa$ is the curvature $(1 / r$ where $r$ is the radius of curvature, positive for a convex shape and negative for a concave shape) and $\gamma$ is the surface tension. This pressure gradient causes the water to migrate from the convex tips to the concave parts between the needles. Eqn (4) also indicates that the liquid pressure declines along the direction of the increasing radius on nanoneedles and the liquid pressure also reduces alongside the direction of decreasing radius between the nanoneedles. This means that the water condensed on the tip of the nanoneedle will be drawn towards the periphery of the nanoneedle base. Similarly, the water deposited around the periphery of the nanoneedle base will be drawn towards the corner region. On the nanoneedle, the radius of tip $r_{\mathrm{t}}$ is visibly much smaller as compared to the radius of periphery $r_{\mathrm{p}}\left(r_{\mathrm{p}}>r_{\mathrm{t}}\right)$ and the radius of the base $r_{\mathrm{b}}$ can be considered much larger as compared to the periphery $\left(r_{\mathrm{b}}>r_{\mathrm{p}}\right.$, see Fig. 9). This indicates that the overall water deposited as a film will be drawn towards the region near the periphery of the nanoneedle by the influence of the pressure gradient. It was interesting to observe that inter-spacing and the angles in nanoneedles were sufficient for continuous removal of water film and avoiding over-flooding which could be the reason for the increase in fog deposition over the surfaces. ${ }^{73}$ Therefore water keeps on depositing on the uncoated nanoneedle surfaces which show very good water collection efficiency as compared to the control uncoated surfaces.
On the surfaces bearing hydrophobic coating, a different type of water collection was observed. On the control hydrophobic smooth copper surface, very small water droplets got deposited on the surface due to the surface being a barrier to the fog flow. This is because of the low surface tension nature of the surface which delays the droplet nucleation. These tiny droplets gradually became bigger in size to eventually merge with the other droplets. After the droplet attained a critical size, it slides off the surface. The critical size of the droplet pinned to a surface under the influence of gravity on a completely vertical surface can be estimated using the following equation: ${ }^{13,71}$

$$
\rho V g=\gamma D\left(\cos \theta_{\mathrm{r}}-\cos \theta_{\mathrm{a}}\right)
$$

Here, $\rho$ is the density of the droplet, $\gamma$ is the surface tension and $V$ is the volume of the drop, $D$ is the diameter of the drop, $\theta_{\mathbf{r}}$ and $\theta_{\mathrm{a}}$ represent the receding and advancing contact angles; and $g$ denotes gravitational force. It is clear from eqn (5) that the volume of the droplet is proportional to the contact angle hysteresis. The surface displayed large droplets during fog harvesting experiments because of its high contact angle hysteresis (refer Table 1). Hence, much time was taken to reach the critical volume of the droplet, hence leading to a very long onset time. On the other hydrophobic control surface (scored $\mathrm{Cu}$ ), a comparatively small contact angle hysteresis along with the small critical size of droplets was evidenced which can be due to the presence of large microstructures induced by scoring with sandpaper. In this case, many small droplets were evidenced on the surface at the initial stage of fog collection experiments which eventually grew in size and coalesced with nearby droplets. When drops coalesce, due to the low adhesion to the silane layer, the excess surface energy can lead to the shedding of droplets from the surface. These droplets were in constant exposure of the fog flow accompanied by the large gap between microstructures (as shown in Fig. 3b) which may have led to detachment of these droplets which get carried away in the fog flow and plausibly explain the delayed droplet growth and lower water collection. Additionally, as the existing droplets continue to grow on the surface, their overall volume kept on expanding. The large and deep microstructures created by scoring lead to the pinning of the water droplets and the droplets could not roll off quickly even after they reached their critical size.

In the case of hydrophobic coated $\mathrm{CuO}$ nanoneedle surface, which displayed further increase in the water harvesting performance, the nanoneedles possess higher surface area and

Table 1 Contact angles $\theta$ (deg) on different surfaces. $\theta_{\mathrm{a}}$ is the advancing contact angle, $\theta_{\mathrm{s}}$ is the static contact angle and $\theta_{\mathrm{r}}$ is the receding contact angle. All the non-coated samples were freshly prepared before the measurements ( 0 days exposure). Standard deviation values of three

\begin{tabular}{|c|c|c|c|c|c|c|}
\hline \multirow[b]{2}{*}{ Sample name } & \multicolumn{3}{|c|}{ No coating } & \multicolumn{3}{|c|}{ Hydrophobic coating } \\
\hline & $\theta_{\mathrm{a}}$ & $\theta_{\mathrm{s}}$ & $\theta_{\mathrm{r}}$ & $\theta_{\mathrm{a}}$ & $\theta_{\mathrm{s}}$ & $\theta_{\mathrm{r}}$ \\
\hline Scored $\mathrm{Cu}$ & $121 \pm 3$ & $101 \pm 5$ & $70 \pm 3$ & $141 \pm 8$ & $130 \pm 9$ & $113 \pm 3$ \\
\hline CuO nanoneedles & - & $7 \pm 2$ & - & $171 \pm 2$ & $165 \pm 1$ & $161 \pm 4$ \\
\hline
\end{tabular}
independent measurements are also included 
have more nucleation sites for fog droplet deposition. The nucleated and coalesced droplets get confined between the high aspect ratio, dense and low energy nanoneedle surface. Due to the high surface area, the probability of the droplets colliding with other droplets increases. Another interesting feature of the nanoneedles arranged in arrays is their irregular tilting throughout the surface as evidenced in Fig. 3c. This makes sure that there is a minimal loss of water droplets from the surface due to the fog flow and the droplets which escape from the tilted nanoneedle surface get trapped by neighboring nanoneedles. Thus, the coalesced droplets in this way reached a critical size and start rolling off the surface rapidly which can be evidenced from the very short onset time and lower droplet mass (refer Fig. 8b). The presence of random microflower-like structures may also aid in the droplet growth by providing pinning sites. Also, we assume that there is optimal space between the nanoneedles which avoids the flooding between the nanoneedles and loss of superhydrophobicity which can arise from any trapped coalesced droplet. This can be confirmed with the repeatability tests conducted on the surfaces (Fig. S7, ESI $\dagger$ ) where the surfaces maintained efficient water collection ability even after 5 fog collection cycles.

It is also noteworthy that the hydrophobic coated $\mathrm{CuO}$ nanoneedle surface collected more amount of water than the hydrophilic $\mathrm{CuO}$ nanoneedle surface, unlike in the previous study $^{73}$ where the hydrophilic conical structures collected more water than the hydrophobic conical structures. This can be because of a phenomenon called "coalescence cascade" where the droplets jump off the surface of water due to a very thin layer of air underneath the droplet. ${ }^{75}$ Since there is formation of a water film on the surface of hydrophilic $\mathrm{CuO}$ nanoneedles and the surface is in a tilted position during the fog collection experiments, the jumping droplets do not bounce back to the water film and get carried away in the fog flow.

\section{Guided transport of the harvested water}

In general, a fog collection phenomenon consists of three stages which include nucleation of fog droplets on the surface, droplet growth and removal of water from the surface. During the last phase of water removal, there is a probability of a significant water loss due to the evaporation, especially when the fog harvesting is taking place at a large scale. In the hydrophilic surfaces, harvested and condensed fog droplets merge to make a film of water over the surface which is more prone to evaporation. On the hydrophobic surfaces, the harvested droplets which roll off the surfaces have a tendency to foul the other nucleating droplets and terminate the nucleation and growth process prematurely. So for the efficient collection of water, it is very important to transport the captured water proficiently. ${ }^{52}$

To achieve efficient water transport, the hydrophilic channels were made with a line width of $1 \mathrm{~mm}$ by employing the milling technique (refer Fig. 1a). For efficient transport of the water during the fog harvesting process, the surfaces were tilted slightly. Fig. 10 shows the overall water collected by the surfaces with channels and without channels. It is clear from the figure that the presence of channels significantly adds to the overall

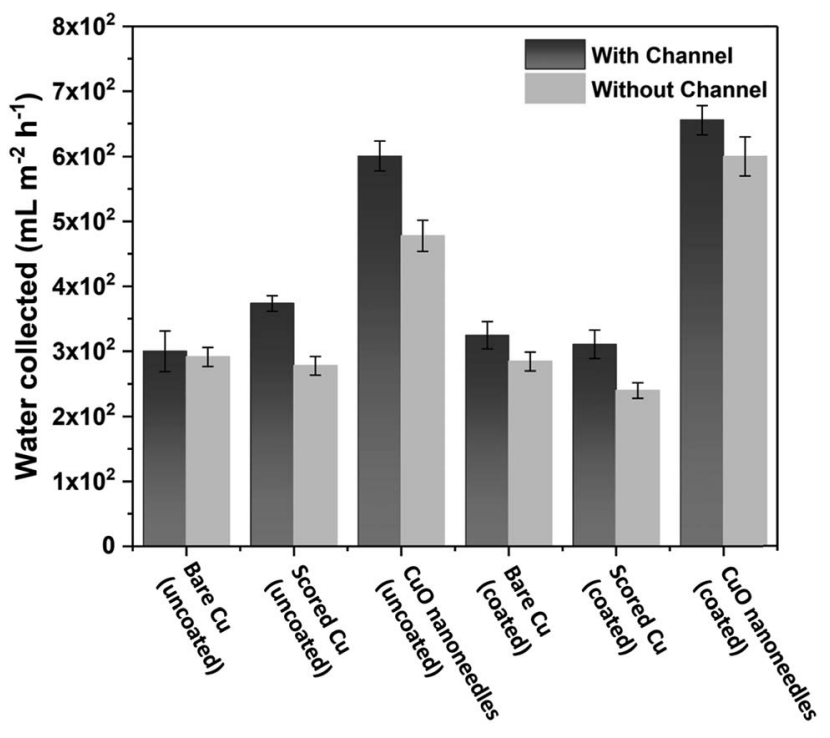

Fig. 10 Comparison of the rates of water collection for surfaces with and without channels.

quantity of water collected over the surfaces. On the hydrophilic surfaces, the harvested water from the nearby areas coalesce with the film and gets transported via the capillary-like system with the aid of gravity (refer Fig. 11a). Such a fog harvesting mechanism is in agreement with the earlier reported simultaneous dropwise and filmwise condensation on structured and patterned wettability surfaces as in the work of Orejon $e t$ al. ${ }^{76}$ and Mahapatra et al. ${ }^{77}$ When the hydrophilic channels are initially filled with water, the water channeling through them is swifter. This is evident from Fig. 7a where the initial water collection is low, displays a cascading pattern and follows a steady flow afterward.

The water transport in the hydrophobic surfaces was different than the surfaces possessing hydrophilic surfaces. A few droplets were observed to roll along the channel while most of the droplets were observed to change their shape and move along the channel. Fig. 11b shows the transport of the water droplets on to the hydrophilic guiding tracks milled on to the hydrophobic surfaces. It can be seen from the figure that the
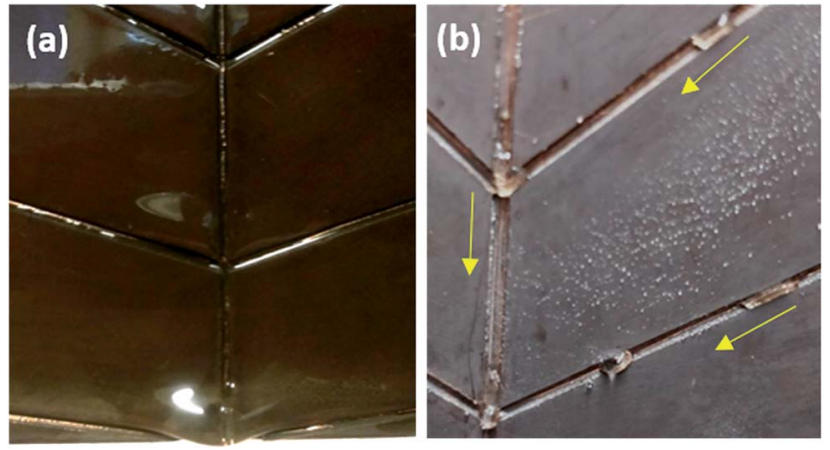

Fig. 11 Guided transport of water via hydrophilic milled channels in (a) $\mathrm{CuO}$ nanoneedles (uncoated) and (b) CuO nanoneedles (coated). 
droplets deposited on the hydrophilic channel display a significant change in the morphology of the drop, elongating along the channel.

To explain why a drop is pinned to the hydrophilic track, consider the change in free energy of the drop when it encounters the track. To keep the argument simple, we will only consider a two-dimensional model of Fig. 12, which shows a schematic of a drop interacting with an infinite length channel, where the channels are hydrophilic and rest of the surface is hydrophobic.

When the contact line advances an infinitesimal amount $\mathrm{d} x$ on the substrate, the surface free energy is increased by $\mathrm{d} E=$ $w \mathrm{~d} x\left(\gamma_{\mathrm{sl}}-\gamma_{\mathrm{sg}}\right)=-w \gamma \mathrm{d} x \cos \theta$, where $w$ is the width of the drop (see Fig. 12) and $\gamma_{\mathrm{sl}}$ and $\gamma_{\mathrm{sg}}$ are the surface energies of the solidliquid and solid-gas interfaces, respectively, and $\gamma$ is the surface tension of the liquid. The free energy associated with the dropgas interfaces is $E=\gamma(w s+2 A)$, where $s$ is the arc length of the liquid-gas interface and accounts for the top interface and $A$ is the cross-sectional area and accounts for the front- and back interfaces. For the total surface free energy of the drop, we have

$$
E=\gamma\left[w\left(s-x_{1} \cos \theta_{1}-x_{2} \cos \theta_{2}\right)+2 A\right]
$$

where $x_{1,2}$ are the wetted perimeters of the channel and the background substrate, respectively, and $\theta_{1,2}$ are their contact angles. Since the channel is hydrophilic $\left(\theta_{1}<90^{\circ} \Rightarrow \cos \theta_{1}>0\right)$, increasing $x_{1}$ decreases the free energy. Similarly, since the channel is hydrophilic $\left(\theta_{2}>90^{\circ} \Rightarrow \cos \theta_{2}<0\right)$, decreasing $x_{2}$ decreases the free energy.

Assuming that the drop has fully wetted the channel $\left(x_{1}=2 h\right.$ $+l$, where $h$ is the height of the channel and $l$ is its length) and dewetted the hydrophobic surface $\left(x_{2}=0\right)$, the contact line is pinned to the edge of the channel and it can be seen that the interface of the drop can thus lower its free energy by increasing $w$, i.e. by elongating along the channel, up to the point where $\mathrm{d} E / \mathrm{d} w=0$. This elongation can be clearly seen in Fig. 12. This explains how the drops coalesce in the open channels due to hydrophilic interactions and get transported as a capillary system.

In summary, the bioinspired surfaces based on $\mathrm{CuO}$ nanoneedle arrays showed good fog collection efficiency per unit area at a consistent rate and at a large scale. The results
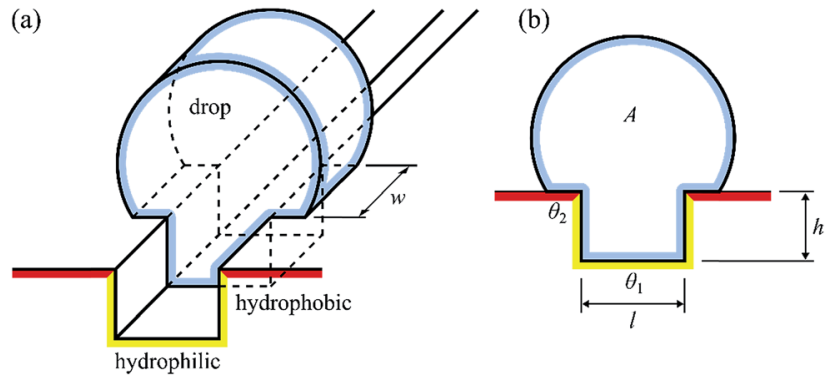

Fig. 12 Two-dimensional model of a drop interacting with an infinite length channel in the case where the channels are hydrophilic and rest of the surface is hydrophobic. displayed in this study are at par with the bioinspired surfaces based on micro-conical arrays ${ }^{\mathbf{1 0 , 1 8 , 1 9 , 4 2 , 5 0 , 7 3 , 7 8 , 7 9}}$ as well as other structures existing in the literature. As reported in the previous studies, the arrangement of the super-hydrophobic and superhydrophilic surfaces together in different orientations and patterns can further enhance the overall water collection ability of the surfaces. ${ }^{\mathbf{1 2 , 2 0 , 5 1}}$ So using these coated and non-coated nanoneedle-based surfaces, the creation of varying wettability patterns in different permutations and combinations to check any variation in the water collection dynamics would be another interesting study. The knowledge and the findings based on these efficient fog-collecting surfaces at a large scale can pave way for the design and improvement of highly efficient surfaces to be utilized in fog-harvesting and other industrial applications.

\section{Conclusion}

In this paper, we demonstrated bioinspired $\mathrm{CuO}$ surfaces possessing nanoneedle-like structures using a simple wet chemical oxidation technique which can be scaled up very easily. The overall fabrication process along with the formation of nanoneedle-like structures has been discussed and the surfaces have been characterized thoroughly. It was observed that the surfaces bearing $\mathrm{CuO}$ nanoneedles show good droplet spreading and can be easily converted to super-hydrophobic surfaces with silane coating. This method of fabrication represents a way of obtaining functional surfaces at a large scale for efficient water harvesting and the overall increase in fog harvesting efficiency observed was $\sim 99 \%$ for non-coated and $\sim 150 \%$ for surfaces with hydrophobic coating when compared to the smooth control samples. In addition, hydrophilic channels were fabricated on to these surfaces using milling for efficient guided water transport. The presence of channels tends to increase the overall water harvesting capability of the surfaces by minimizing the risk of evaporation and fouling by already captured and departing droplets. The presence of channels further enhanced the water collection efficiency by $\sim 9 \%$ when compared to the surfaces without channels. To support the experimental data, a 2-dimensional model was proposed to show transportation of droplets adsorbed in the open channels as a capillary system due to the hydrophilic interactions. The insights gained by these results can be beneficial towards the design and fabrication of efficient fogharvesting devices. Apart from water harvesting, these surfaces can also be utilized in different practical fields such as catalysis, electronics, and applications related to heat transfer.

\section{Conflicts of interest}

There are no conflicts to declare.

\section{Acknowledgements}

This work was supported by the Academy of Finland (grants: \#299087 and \#292477). All authors are grateful for the support 
from Tampere Microscopy Center for characterization of the surfaces.

\section{References}

1 M. M. Mekonnen and A. Y. Hoekstra, Sci. Adv., 2016, 2, e1500323.

$2 \mathrm{~W}$. H. Organization and Unicef, Progress on sanitation and drinking water-2015 update and MDG assessment, World Health Organization, 2015.

3 A. du Plessis, in Water as an Inescapable Risk, Springer, 2019, pp. 13-25.

4 R. Clarke, Water: the international crisis, Routledge, 2013.

5 J. Yao and G. Yang, J. Mater. Chem. A, 2018, 6, 3869-3876.

6 O. Klemm, R. S. Schemenauer, A. Lummerich, P. Cereceda, V. Marzol, D. Corell, J. van Heerden, D. Reinhard, T. Gherezghiher, J. Olivier, P. Osses, J. Sarsour, E. Frost, M. J. Estrela, J. A. Valiente and G. M. Fessehaye, Ambio, 2012, 41, 221-234.

7 L. Zhang, J. Wu, M. N. Hedhili, X. Yang and P. Wang, J. Mater. Chem. A, 2015, 3, 2844-2852.

8 K.-C. Park, S. S. Chhatre, S. Srinivasan, R. E. Cohen and G. H. McKinley, Langmuir, 2013, 29, 13269-13277.

9 Y. Wang, L. Zhang, J. Wu, M. N. Hedhili and P. Wang, J. Mater. Chem. A, 2015, 3, 18963-18969.

10 C. Li, Y. Liu, C. Gao, X. Li, Y. Xing and Y. Zheng, ACS Appl. Mater. Interfaces, 2019, 11(4), 4507-4513.

11 B. White, A. Sarkar and A.-M. Kietzig, Appl. Surf. Sci., 2013, 284, 826-836.

12 R. Hu, N. Wang, L. Hou, Z. Cui, J. Liu, D. Li, Q. Li, H. Zhang and Y. Zhao, J. Mater. Chem. A, 2019, 7, 124-132.

13 V. Sharma, D. Orejon, Y. Takata, V. Krishnan and S. Harish, ACS Sustainable Chem. Eng., 2018, 6, 6981-6993.

14 W. Shi, M. J. Anderson, J. B. Tulkoff, B. S. Kennedy and J. B. Boreyko, ACS Appl. Mater. Interfaces, 2018, 10, 1197911986.

15 B. Bhushan, in Biomimetics, Springer, 2018, pp. 665-701.

16 X. Dai, N. Sun, S. O. Nielsen, B. B. Stogin, J. Wang, S. Yang and T.-S. Wong, Sci. Adv., 2018, 4, eaaq0919.

17 V. Sharma, M. Sharma, S. Kumar and V. Krishnan, Flora, 2016, 224, 59-65.

18 Y. Peng, Y. He, S. Yang, S. Ben, M. Cao, K. Li, K. Liu and L. Jiang, Adv. Funct. Mater., 2015, 25, 5967-5971.

19 J. Ju, H. Bai, Y. Zheng, T. Zhao, R. Fang and L. Jiang, Nat. Commun., 2012, 3, 1247.

20 T. Nørgaard and M. Dacke, Front. Zool., 2010, 7, 23.

21 Y. Zheng, H. Bai, Z. Huang, X. Tian, F.-Q. Nie, Y. Zhao, J. Zhai and L. Jiang, Nature, 2010, 463, 640.

22 H. Dong, N. Wang, L. Wang, H. Bai, J. Wu, Y. Zheng, Y. Zhao and L. Jiang, ChemPhysChem, 2012, 13, 1153-1156.

23 H. Zhu, Z. Guo and W. Liu, Chem. Commun., 2016, 52, 38633879.

24 H. Zhu and Z. Guo, Chem. Commun., 2016, 52, 6809-6812.

25 M. A. K. Azad, W. Barthlott and K. Koch, Langmuir, 2015, 31, 13172-13179.

26 Y. Wang, X. Wang, C. Lai, H. Hu, Y. Kong, B. Fei and J. H. Xin, ACS Appl. Mater. Interfaces, 2016, 8, 2950-2960.
27 H. G. Andrews, E. A. Eccles, W. C. E. Schofield and J. P. S. Badyal, Langmuir, 2011, 27, 3798-3802.

28 Y. Lu, L. Yu, Z. Zhang, S. Wu, G. Li, P. Wu, Y. Hu, J. Li, J. Chu and D. Wu, RSC Adv., 2017, 7, 11170-11179.

29 M. A. K. Azad, T. Krause, L. Danter, A. Baars, K. Koch and W. Barthlott, Langmuir, 2017, 33, 5555-5564.

30 J. Ju, K. Xiao, X. Yao, H. Bai and L. Jiang, Adv. Mater., 2013, 25, 5937-5942.

31 Y. Jiang, S. Savarirayan, Y. Yao and K.-C. Park, Appl. Phys. Lett., 2019, 114, 83701.

32 X. Chen, J. Chen, X. Ouyang, Y. Song, R. Xu and P. Jiang, Langmuir, 2017, 33, 6701-6707.

33 X. Yan, Z. Huang, S. Sett, J. Oh, H. Cha, L. Li, L. Feng, Y. Wu, C. Zhao, D. Orejon, F. Chen and N. Miljkovic, ACS Nano, 2019, 13, 4160-4173.

34 H. K. Raut, A. S. Ranganath, A. Baji and K. L. Wood, Appl. Surf. Sci., 2019, 465, 362-368.

35 D. Orejon, A. Askounis, Y. Takata and D. Attinger, ACS Appl. Mater. Interfaces, 2019, 11, 24735-24750.

36 H. Bai, L. Wang, J. Ju, R. Sun, Y. Zheng and L. Jiang, Adv. Mater., 2014, 26, 5025-5030.

37 S. H. Lee, J. H. Lee, C. W. Park, C. Y. Lee, K. Kim, D. Tahk and M. K. Kwak, International Journal of Precision Engineering and Manufacturing-Green Technology, 2014, 1, 119-124.

38 M. Cao, J. Xiao, C. Yu, K. Li and L. Jiang, Small, 2015, 11, 4379-4384.

39 K.-C. Park, P. Kim, A. Grinthal, N. He, D. Fox, J. C. Weaver and J. Aizenberg, Nature, 2016, 531, 78.

40 M. Gürsoy, M. T. Harris, A. Carletto, A. E. Yaprak, M. Karaman and J. P. S. Badyal, Colloids Surf., A, 2017, 529, 959-965.

41 J. Ju, Y. Zheng and L. Jiang, Acc. Chem. Res., 2014, 47, 23422352.

42 M. Cao, J. Ju, K. Li, S. Dou, K. Liu and L. Jiang, Adv. Funct. Mater., 2014, 24, 3235-3240.

43 C. Lv, P. Hao, X. Zhang and F. He, ACS Nano, 2015, 9, 1231112319.

44 R. Wen, S. Xu, D. Zhao, Y.-C. Lee, X. Ma and R. Yang, ACS Appl. Mater. Interfaces, 2017, 9, 44911-44921.

45 M. E. R. Shanahan, Langmuir, 2011, 27, 14919-14922.

46 J. Seo, S. Lee, J. Lee and T. Lee, ACS Appl. Mater. Interfaces, 2011, 3, 4722-4729.

47 H. Mertaniemi, R. Forchheimer, O. Ikkala and R. H. A. Ras, Adv. Mater., 2012, 24, 5738-5743.

48 V. Sharma, R. Balaji and V. Krishnan, Biomimetics, 2018, 3, 7. 49 J. S. Price, Boundary-Layer Meteorol., 1991, 57, 391-406.

50 J. Ju, X. Yao, S. Yang, L. Wang, R. Sun, Y. He and L. Jiang, Adv. Funct. Mater., 2014, 24, 6933-6938.

51 D. Xing, F. Wu, R. Wang, J. Zhu and X. Gao, ACS Appl. Mater. Interfaces, 2019, 11(7), 7553-7558.

52 H. Bai, C. Zhang, Z. Long, H. Geng, T. Ba, Y. Fan, C. Yu, K. Li, M. Cao and L. Jiang, J. Mater. Chem. A, 2018, 6, 20966-20972.

53 Y. Xing, W. Shang, Q. Wang, S. Feng, Y. Hou and Y. Zheng, ACS Appl. Mater. Interfaces, 2019, 11(11), 10951-10958.

54 K. Lahtonen, M. Lampimäki, P. Jussila, M. Hirsimäki and M. Valden, Rev. Sci. Instrum., 2006, 77, 83901. 
55 M. Hirsimäki, M. Lampimäki, K. Lahtonen, I. Chorkendorff and M. Valden, Surf. Sci., 2005, 583, 157-165.

56 J. H. Scofield, J. Electron Spectrosc. Relat. Phenom., 1976, 8, 129-137.

57 E. Kostal, S. Stroj, S. Kasemann, V. Matylitsky and M. Domke, Langmuir, 2018, 34, 2933-2941.

58 A. R. Parker and C. R. Lawrence, Nature, 2001, 414, 33.

59 B. Chang, A. Shah, Q. Zhou, R. H. A. Ras and K. Hjort, Sci. Rep., 2015, 5, 14966.

60 Y. Jin, L. Zhang and P. Wang, Global Challenges, 2017, 1, 1700019.

61 S. Wang, M. Liu, Y. Feng, Y. Bu, S. H. Huynh, T. W. Ng, F. Gu, A. Yu and X. Jiang, J. Mater. Chem. A, 2017, 5, 21422-21428.

62 W. Zhang, X. Wen, S. Yang, Y. Berta and Z. L. Wang, Adv. Mater., 2003, 15, 822-825.

63 A. Chaudhary and H. C. Barshilia, J. Phys. Chem. C, 2011, 115, 18213-18220.

64 L. Schlur, K. Bonnot and D. Spitzer, RSC Adv., 2015, 5, 60616070.

65 D. P. Singh, A. K. Ojha and O. N. Srivastava, J. Phys. Chem. C, 2009, 113, 3409-3418.

66 P. Zhang, Y. Maeda, F. Lv, Y. Takata and D. Orejon, ACS Appl. Mater. Interfaces, 2017, 9, 35391-35403.

67 Y. Shingaya and M. Ito, J. Electroanal. Chem., 1999, 467, 299306.
68 D. Quéré, Annu. Rev. Mater. Res., 2008, 38, 71-99.

69 Z. Wang, J. Zhao, A. Bagal, E. C. Dandley, C. J. Oldham, T. Fang, G. N. Parsons and C.-H. Chang, Langmuir, 2016, 32, 8029-8033.

70 V. Sharma, S. Kumar, A. Jaiswal and V. Krishnan, ChemistrySelect, 2017, 2, 165-174.

71 A. Carre and M. E. R. Shanahan, J. Adhes., 1995, 49, 177-185.

72 D. Orejon, K. Sefiane and M. E. R. Shanahan, Langmuir, 2011, 27, 12834-12843.

73 H. Cho, B. Park, M. Kim, S. Lee and W. Hwang, J. Mater. Chem. A, 2017, 5, 25328-25337.

74 R. L. Webb and N.-H. Kim, Principles Enhanced Heat Trans, Garland Science, 2004.

75 S. T. Thoroddsen and K. Takehara, Phys. Fluids, 2000, 12, 1265-1267.

76 D. Orejon, O. Shardt, N. S. K. Gunda, T. Ikuta, K. Takahashi, Y. Takata and S. K. Mitra, Int. J. Heat Mass Transfer, 2017, 114, 187-197.

77 P. S. Mahapatra, A. Ghosh, R. Ganguly and C. M. Megaridis, Int. J. Heat Mass Transfer, 2016, 92, 877-883.

78 T. Xu, Y. Lin, M. Zhang, W. Shi and Y. Zheng, ACS Nano, 2016, 10, 10681-10688.

79 Q. Wang, Q. Meng, M. Chen, H. Liu and L. Jiang, ACS Nano, 2014, 8, 8757-8764. 